\title{
A CALENDAR OF LETTERS WRITTEN TO WHITMAN
}

(Revised 1991)

This Calendar includes extant letters written to WW. The following information appears in the entries: (1) the date; (2) the name of the correspondent, sometimes with a brief identification in order to indicate the nature of the correspondence; (3) the location of the letter, if known; and (4) appearances in print, if any. All letters in the Calendar unless otherwise indicated are in the Charles E. Feinberg Collection at the Library of Congress. Those letters marked LC are in other Whitman collections in the Library of Congress. Abbreviations are explained in the table of abbreviations on pp. 6-7.

1840

June 27. From Abraham Paul Leech. Lc. August 10. From Abraham Paul Leech. LC.

\section{2(?)}

March 31. From Aaron Smith.

1855

July 21. From Ralph Waldo Emerson. CT: Corr, 1:41.

\section{6}

April 21(?). From Fanny Fern (Mrs. James Parton). ст: The American Book Collector, 11 (May 1961): 8-9.

June 7. From S. R. Wells.

June 29. From George I. Storms, a friend. September 16. From Charles S. Keyser.

\section{7}

June 24. From Sarah Tyndale.

July 1. From Sarah Tyndale.

\section{0}

February 10. From Thayer \& Eldridge. cT: Allen, 236-37.

February 27. From Thayer \& Eldridge.
March 2. From Thayer \& Eldridge.

March 6. From Ticknor \& Fields, for The Atlantic Monthly. Location unknown. CT: Traubel, 4:77.

March 7. From Thayer \& Eldridge.

March 9. From Thayer and Eldridge.

March 19. From Fred Vaughan, a friend. Ст: Shively (1), 41-42.

March 21. From Charles Hine.

March 21. From Fred Vaughan. ст: Shively (1), 42.

March 27. From Henry Clapp Jr. ст: Traubel, 1:236-37.

March 27. From Fred Vaughan. Facsimile: Shively (1), [44-45], 43, 46.

March 30. From Louisa Van Velsor Whitman. Trent.

April 3. From Thomas Jefferson Whitman. ст: Berthold and Price, 13-15.

April 6. From O. K. Sammis, a friend.

April 9. From Fred Vaughan. ст: Shively (1), 46.

April 16. From Thomas Jefferson Whitman. ст: Berthold and Price, 15-17.

April 23. From Frederick Baker.

April 30. From Fred Vaughan. ст: Shively (1), 46-47.

May 3. From Louisa Van Velsor Whitman. Trent.

May 14. From Henry Clapp Jr. ст: Traubel, 2:375-76.

May 17. From Fred Vaughan. ст: Shively (1), 47 (misdated May 7). 
May 18. From Charles L. Heyde. Trent. Ст: Gohdes and Silver, 215-16.

May 21. From Fred Vaughan. ст: Shively (1), 47.

May 24. From Thayer \& Eldridge.

June 5. From Thayer \& Eldridge.

June 14. From Thayer \& Eldridge.

June 25. From James Redpath. Ст: Traubel, 3:460.

July 11. From Susan Garnet Smith, an admirer. Location unknown. Ст: Traubel, 4:312-13.

July 27. From Thayer \& Eldridge.

August 16. From Wilhelmina Walton.

August 17. From Thayer \& Eldridge.

October 11. From Thayer \& Eldridge.

October 15. From Thayer \& Eldridge.

December 1. From Thayer \& Eldridge.

December 5. From Thayer \& Eldridge.

\section{1}

February 6. From Harvey Jewell and C. S. Kendall, lawyers.

April 19. From W. W. Thayer.

October 10. From the Editors, The Atlantic Monthly. ст: Traubel, 2:213.

\section{2}

January 8. From Lieutenant Silas S. Soule. ст: Shively (2), 187.

March 12. From Lieutenant Silas S. Soule. ст: Shively (2), 187-88.

March 25. From Ellen Eyre. ст: Allen, 279.

May 2. From Fred Vaughan. ст: Shively (1), 48.

August 31-September 6. From Thayer \& Eldridge.

[Summer 1862]. From Lieutenant Silas S. Soule. ст: Shively (2), 188-89.

September 25. From Theodore Rich. LC.

December 19. From Thomas Jefferson Whitman. ст: Berthold and Price, 1719.

December. From Hannah Heyde. Trent.

\section{3}

January 1-2. From Thomas Jefferson Whitman. ст: Berthold and Price, 1921.
January 12. From Ralph Waldo Emerson. ст: Corr., 1:66.

January 13. From George Washington Whitman. Trent. ст: Loving, 80-81.

January 13. From Thomas Jefferson Whitman. ст: Berthold and Price, 2224.

January 15(?). From George Wood.

January 26. From Moses Lane.

February 1. From George Washington Whitman. Trent. ст: Loving, 83.

February 6. From Thomas Jefferson Whitman. ст: Berthold and Price, 2426.

February 10. From Thomas Jefferson Whitman. ст: Berthold and Price, 2628.

February 12. From George Washington Whitman. Trent. ст: Loving, 86-87.

February 12. From Thomas Jefferson Whitman. Berthold and Price, 29-31.

February 25. From George Washington Whitman. Trent. Ст: Loving, 88-89.

February 25(?). From John Swinton. ст: Traubel, 1:416.

February 26. From Harper's Weekly.

March 3. From Thomas Jefferson Whitman. ст: Berthold and Price, 31-34.

March 9. From Thomas Jefferson Whitman. ст: Berthold and Price, 34-35.

March 10. From Justus F. Boyd, soldier. Hanley. ст: Shively (2), 111.

March 10. From James Redpath. Location unknown. Ст: Donaldson, 143.

March 12. From Thomas Jefferson Whitman. ст: Berthold and Price, 35-36.

March 19. From Louisa Van Velsor Whitman. Trent.

March 19. From Thomas Jefferson Whitman. Ст: Berthold and Price, 36 (note to mother's letter).

March 21. From Thomas Jefferson Whitman. Ст: Berthold and Price, 36-40.

March 21. From Charles S. Kingsley, a New York friend. Berg. ст: Shively (2), 153.

March. From Louisa Van Velsor Whitman. Trent.

April 2. From Thomas Jefferson Whitman. Ст: Berthold and Price, 40-42.

April 3. From Thomas Jefferson Whitman. Ст: Berthold and Price, 42-43. 
April 5. From Will W. Wallace, soldier. ст: $M L Q, 10$ (1949), 92-93; Shively (2), 208-10.

[April 6]. From Thomas Jefferson Whitman. Ст: Berthold and Price, 44-45.

April 11. From Thomas Jefferson Whitman. ст: Berthold and Price, 45-48.

April 13. From Thomas Jefferson Whitman. CT: Berthold and Price, 48.

April 20. From Thomas Jefferson Whitman. ст: Berthold and Price, 49-50.

April 25. From Thomas Jefferson Whitman. ст: Berthold and Price, 50-52.

April 26. From a friend of Thomas P. Sawyer, soldier. Berg. ст: Shively (1), 74-75.

April 27. From Justus F. Boyd. Hanley. Ст: Shively (2), 112.

April 29. From Fred B. McReady, soldier. Berg.

April 30. From Moses Lane. LC.

May 1. From John F. S. ("Fred") Gray. Hanley. ст: Shively (2), 147-49.

May 2. From Thomas Jefferson Whitman. ст: Berthold and Price, 52-54.

May 2. From Moses Lane. LC.

May 5. From James Redpath. Historical Society of Pennsylvania. PT: Donaldson, 144.

May 7. From Will W. Wallace. ст: $M L Q$, 10 (1949), 93-94; Shively (2), 210-11.

May 9. From Thomas Jefferson Whitman. Ст: Berthold and Price, 54-55.

May 12. From Thomas Jefferson Whitman. ст: Berthold and Price, 56-57.

May 14. From Justus F. Boyd. Berg. ст: Shively (2), 112-13.

May 27. From Thomas Jefferson Whitman. ст: Berthold and Price, 57-59.

May 27. From Moses Lane.

June 5. From John J. Barker, soldier. Hanley:Texas. ст: Shively (2), 108.

June 13. From Thomas Jefferson Whitman. ст: Berthold and Price, 59-61.

June 19. From John J. Barker. Hanley. ст: Shively (2), 109-10.

July 1. From Will W. Wallace. Berg. ст: $M L Q, 10$ (1949), 94-95; Shively (2), 211-12.

July 7. From Thomas Jefferson Whitman. Ст: Berthold and Price, 62.

July 8. From Thomas Jefferson Whitman. ст: Berthold and Price, 63.
July 10. From Lewis K. Brown, soldier. Hanley. ст: The Long Islander, May 24, 1962; Shively (2), 117 (misdated July 20).

July 19. From Thomas Jefferson Whitman. ст: Berthold and Price, 63-67.

After July 23(?), From Louisa Van Velsor Whitman. Trent.

July 24. From Alvah H. Small, soldier. Berg. ст: Shively (2), 179-80.

July 27. From Lewis K. Brown. Hanley: Texas. CT: The Long Islander, May 24, 1962; Shively (2), 118.

July 31. From William E. Vandemark, soldier. Hanley. ст: Shively (2), 204.

August 4. From Thomas Jefferson Whitman. ст: Berthold and Price, 67-69.

August 10. From Lewis K. Brown. Berg. Ст: Shively (1), 76-77.

August 16-25(?). From Louisa Van Velsor Whitman. Trent.

August 17. From William E. Vandemark. Hanley. ст: Shively (2), 204-5.

August 18. From Mary A. Babbitt (for Caleb H. Babbitt, soldier). Berg. Ст: Shively (2), 103.

August 22. From Lewis K. Brown. Hanley. ст: Shively (2), 119-20.

August 27. From S. B. and R. O. Haskell, parents of soldier. Formerly in Hanley Collection.

August 31. From C. L. Scott, soldier. Berg. ст: Shively (2), 178.

September 3(?). From Louisa Van Velsor Whitman. Trent.

[About September 3]. From Louisa Van Velsor Whitman. Trent.

September 5. From Thomas Jefferson Whitman. ст: Berthold and Price, 6971.

September 5, evening. From Thomas Jefferson Whitman. CT: Berthold and Price, 71-73.

September 6. From Mary A. Babbitt, sister of soldier. Berg. ст: Shively (2), 1034.

September 17. From Bethuel Smith, soldier. ст: Shively (2), 181.

September 17 later. From Bethuel Smith. CT: Shively (2), 181-83.

September 18. From Caleb H. Babbitt. Berg. ст: Shively' (2), 104-5. 
September 21. From Dr. Le Baron Russell. Hanley. ст: Donaldson, 145-46.

September 22. From Thomas Jefferson Whitman. ст: Berthold and Price, 7374.

September 24. From Thomas Jefferson Whitman. ст: Berthold and Price, 7476.

September 25. From Fanny Van Nostrand. LC.

September 28. From Bethuel Smith. CT: Shively (2), 182.

October 1. From Margaret S. Curtis. Hanley. ст: Donaldson, 147.

October 4. From Dr. Le Baron Russell. Hanley. ст: Donaldson, 147-48.

October 6. From Dr. Le Baron Russell. Hanley.

October 6. From Hannah E. Stevenson. Hanley. ст: Donaldson, 150.

October 7. From Thomas Jefferson Whitman. ст: Berthold and Price, 76-77.

October 8. From Thomas Jefferson Whitman. ст: Berthold and Price, 78-79.

October 8. From James Redpath. Historical Society of Pennsylvania.

October 13. From Bethuel Smith.

October 13. From Julia Stillwell, sister of soldier. Berg. ст: Shively (2), 190.

October 14. From James Redpath. Location unknown. PT: Donaldson, 151.

October 15. From Thomas Jefferson Whitman. ст: Berthold and Price, 7980.

October 18. From Caleb H. Babbitt. Berg. ст: Shively (2), 105-6.

October 20. From John and Margaret Stillwell, parents of soldier. Berg. СT: Shively (2), 191.

October 21(?). From Louisa Van Velsor Whitman. Trent.

October 22. From Thomas Jefferson Whitman. ст: Berthold and Price, 8082.

October 24. From Dr. Le Baron Russell. Hanley.

October 25. From Margaret Stillwell. ст: Shively (2), 192-93.

October 26. From Louisa Van Velsor Whitman. Trent.

October 26. From S. H. Childs (for Caleb H. Babbitt). Berg. ст: Shively (2), 106.
October 27. From James Redpath. Syracuse. ст: Traubel, 4:418.

October (?). From Louisa Van Velsor Whitman. Trent. ст: Gohdes and Silver, 184-87.

November 2. From William E. Vandemark. Hanley. ст: Shively (2), 205.

November 5: From Lewis K. Brown. Hanley. ст: Shively (2), 120-21.

November 7. From Elijah Douglass Fox, soldier. Hanley. ст: The Long Islander, May 24, 1962; Shively (2), 143-44.

November 8. From Dr. Le Baron Russell. Hanley. ст: Donaldson, 151.

November 10. From Elijah Douglass Fox. Lion:NYPL. Ст: The Long Islander, May 24, 1962; Shively (1), 79-80.

November 10. From Ellen M. O'Connor.

November 11. From William H. McFarland, soldier. Hanley. ст: Shively (2), 157-58.

November 13-14. From Lewis K. Brown. Hanley. ст: Shively (2), 123-26.

November 21. From Livingston J. Brooks, soldier. Berg. ст: Shively (2), 115-16.

November 21. From Ellen M. O'Connor. CT: Traubel, 3:524-25.

November 24. From Ellen M. O'Connor.

December 3. From Thomas Jefferson Whitman. ст: Berthold and Price, 8283.

December 4. From Louisa Van Velsor Whitman. Trent. ст: Gohdes and Silver, 187-90.

December 7. From William E. Vandemark. Hanley. ст: Shively (2), 205-6.

December 9. From Elijah Douglass Fox. LC. Ст: The Long Islander, May 24, 1962. PT: Shively (2), 145.

December 14. From Benjamin Penhallow Shillaber. Ст: Traubel, 2:96-97 (misdated December 10).

December 15(?). From Louisa Van Velsor Whitman. Trent.

December 15. From Thomas Jefferson Whitman. ст: Berthold and Price, 8487.

December 16. From Bethuel Smith. ст: Shively (2), 182-83.

December 16. From William E. Vandemark. Berg. ст: Shively (2), 206-7. 
December 21. From John T. Trowbridge. Ст: Traubel, 2:109.

December 22. From Livingston J. Brooks. Hanley. ст: Shively (2), 116.

December 22. From Alonzo S. Bush. Yale. ст: Shively (1), 81-82.

December 21-23. From Martha Whitman. Ohio Wesleyan. ст: Waldon, 31-36.

December 24. From Elijah Douglass Fox. Hanley. ст: The Long Islander, May 24, 1962; Shively (2), 145.

December 25. From William E. Vandemark. Berg. ст: Shively (2), 207.

December 28. From Thomas Jefferson Whitman. ст: Berthold and Price, 8792.

December 28. From Margaret Stillwell. Berg. ст: Shively (2), 193-94.

December 29. From William E. Vandemark. Berg. ст: Shively (2), 207-8.

December 30. From John T. Trowbridge. Location unknown. ст: Traubel, 4:29091.

\section{4}

January 8. From Thomas Jefferson Whitman. ст: Berthold and Price, 92.

January 9. From William H. Millis Sr., father of soldier. Berg. ст: Shively (2), 158.

January 21. From Thomas P. Sawyer. Berg. ст: Shively (1), 82-83.

February 2. From Thomas B. Neat, soldier. Berg. ст: Shively (2), 166-67.

February 11. From Alonzo S. Bush. Hanley. ст: Shively (2), 128-29.

February 12. From John T. Trowbridge. ст: Traubel, 2:524.

February 20. From Andrew J. Liebenan, soldier. Hanley: Texas. ст: Shively (2), 156.

February 28. From Bethuel Smith. ст: Shively (2), 183.

March 5. From Alfred L. Larr, soldier. Hanley. ст: Shively (2), 129.

March 7. From Alonzo S. Bush. Hanley: Texas. ст: Shively (2), 129-30.

March 9. From W. A. Jellison, soldier. Hanley. ст: Shively (2), 152.

March 11. From Thomas Jefferson Whitman. ст: Berthold and Price, 93-95.
March 19. From Thomas Jefferson Whitman. ст: Berthold and Price, 95-96.

March 28. From Rodney R. Worster, soldier. Berg. ст: Shively (2), 229-30.

April 7. From William E. Vandemark. LC. ст: Charles I. Glicksberg, Walt Whitman and the Civil War (1933), 156n.

April 16. From George Washington Whitman. Trent. ст: Loving, 115-17.

April 21. From Mrs. George W. Briggs. Hanley. ст: Donaldson, 151-52; New England Quarterly, 59 (1986):546.

April 29. From George Washington Whitman. Trent. ст: Loving, 117-18.

April 30. From Ruben Farwell, soldier. Trent. ст: Shively (2), 135.

May 5. From Ruben Farwell. Trent. ст: Shively (2), 135-36.

May 7. From Isaac Linensparger, soldier. Berg. ст: Shively (2), 155.

[May 7]. From John F. S. Gray. Berg. CT: Shively (2), 150.

May 9. From Helen S. Cunningham, sister of soldier. Hanley. ст: Shively (2), 131-32.

May 10. From Ruben Farwell. Trent. ст: Shively (2), 136.

May 14. From Aaron Smith, soldier. Berg.

May 23. From W. E. Worthen.

May 30. From Charles H. Harris, soldier. Berg. ст: Shively (2), 150-51.

June 1. From Justus F. Boyd. Hanley. CT: Shively (2), 113.

June 8. From Ruben Farwell. Trent. cT: Shively (2), 138.

June 11. From Helen S. Cunningham. Berg. ст: Shively (2), 134-35.

June 16. From Ruben Farwell. Trent. CT: Shively (2), 140-41.

June 28. From John Burroughs. Feinberg:LC.

July 2. From William D. O'Connor. Location unknown. ст: Traubel, 4:366-68.

July 5. From Ellen M. O'Connor. Feinberg:LC.

July 5. From James S. Stillwell. Berg. ст: Shively (2), 194.

July 6. From Lewis K. Brown. Berg. ст: Shively (1), 83.

July 13. From Aaron Smith. Berg.

July 14. From Elijah Douglass Fox. Berg. Ст: Shively (1), 84 . 
July 18. From Lewis K. Brown. Berg. ст: The Long Islander, May 24, 1962: Shively (1), 85-86.

July 18. From Ellen $M$. O'Connor.

July 24. From Ellen M. O'Connor.

August 2. From John Burroughs. ст: Traubel, 2:548-49.

August 13. From William D. O'Connor. ст: Traubel, 3:337-40.

August 18. From Ellen M. O'Connor. Feinberg:LC.

August 29. From Christopher and Maria Smith, parents of soldier. ст: Shively (2), 183-84.

August 30. From Bethuel Smith. ст: Shively (2), 184.

September 2. From James S. Stillwell. Berg. ст: Shively (2), 195.

September 5. From Lewis K. Brown. Berg. ст: Shively (1), 86-88.

September 5. From Joseph Harris, soldier. Berg. ст: Shively (2), 151.

September 17. From Lizzie H. Smith, cousin of Milton S. Roberts, soldier. Berg.

September 18. From Justus F. Boyd. Berg. ст: Shively (2), 114.

September 27. From James S. Stillwell. Berg. ст: Shively (2), 195.

October 2. From Ruben Farwell. Trent. ст: Shively (2), 138.

October 17. From Hannah Heyde. Lc.

October 18. From Lieutenant William E. Babcock. Berg.

October 21. From William Mullery, father of soldier. Berg. ст: Shively (2), 161-62.

October 22. From Bethuel Smith.

November 7. From Ruben Farwell. Trent. ст: Shively (2), 139.

November 21. From Ruben Farwell. Trent. cr: Shively (2), 139-40.

November 30. From Ellen M. O'Connor.

December 12. From Lieutenant William E. Babcock. Berg.

December 25. From Lieutenant William E. Babcock. Berg.

December 2[6]. From Jesse Mullery. Berg. ст: Shively (2), 162-64 (dated December 24).

December 30. From William D. O'Connor. ст: Traubel, 2:400-3.

\section{5}

January 4. From Dana F. Wright. Berg.

January 6. From John T. Trowbridge. ст: Traubel, 2:292-93.

January 11. From Herman Storms, soldier. Berg. ст: Shively (2), 196.

January 12. From William T. Otto. Lion. ст: Traubel, 3:470-71.

January 12. From William H. Millis, soldier. Berg. ст: Shively (2), 159.

January 19. From Ellen M. O’Connor.

January 21. From Lieutenant William E. Babcock.

January 21. From Aaron Smith.

January 23. From Jesse Mullery. Berg. ст: Shively (2), 164.

January 24. From William T. Otto. Lion. Ст: Traubel, 3:471.

January 26. From Thomas Jefferson Whitman. ст: Berthold and Price, 9799.

January 26. From Christopher and Maria Smith. ст: Shively (2), 184-85.

January 31. From Thomas Jefferson Whitman. ст: Berthold and Price, 99102.

February 3. From Thomas Jefferson Whitman. ст: Berthold and Price, 1025.

February 5. From John Swinton. ст: Traubel, 2:426.

February 7. From Thomas Jefferson Whitman. ст: Berthold and Price, 1057.

February 16. From Colonel Julius W. Mason. Yale.

February 16. From Colonel Elliott F. Shepard.

February 24(?). From Louisa Van Velsor Whitman. Trent. PT: Allen, 328.

February 28. From Captain William Cook. ст: Traubel, 3:202-3.

March 5. From Louisa Van Velsor Whitman. Trent. ст: Gohdes and Silver, 190-92.

March 7. From Celie M. Burr, an admirer.

April 22. From Peter Eckler.

April 26. From Peter Eckler.

May 1. From Peter Eckler.

May 3. From Jesse Mullery. Berg. ст: Shively (2), 165 . 
May 4. From Thomas Jefferson Whitman. ст: Berthold and Price, 108-9.

May 4. From Peter Eckler.

May 11. From William T. Otto. Lion. CT: Traubel, 3:471.

May 14. From Thomas Jefferson Whitman. ст: Berthold and Price, 109-11.

May 26. From Milford C. Reed. Yale. ст: Shively (2), 172.

June 3(?). From Louisa Van Velsor Whitman. Trent.

June 4. From Thomas Jefferson Whitman. CT: Berthold and Price, 111-12.

June 11. From Jesse Mullery. Berg. CT: Shively (2), 166.

June 18. From Kate Richardson, an admirer.

June 24. From Nicholas D. Palmer.

June 30. From James Harlan. Lion. ст: Traubel, 3:471.

July 14. From George Washington Whitman. Trent. Ст: Gohdes and Silver, 182; Loving, 136.

July 16. From Thomas Jefferson Whitman. Ст: Berthold and Price, 113-15.

July 17. From William Stewart, soldier. Yale. ст: Shively (2), 190.

July 30. From A. Van Rensellaer. Lion. Facsimile: William E. Barton, Abraham Lincoln and Walt Whitman (1928), 98. CT: Traubel, 3:178-79.

August 7. From Alfred Pratt, soldier. ст: Shively (2), 167.

August 8. From Louisa Van Velsor Whitman. Trent.

August 9. From Anson Ryder Jr., soldier. CT: Shively (2), 174-75.

August 23. From William F. Bainbridge, soldier. Yale. ст: Shively (2), 108.

August 25. From Anson Ryder, Jr. Ст: Shively (2) 175-76.

August 29. From Louisa Van Velsor Whitman. Trent.

September 5. From Louisa Van Velsor Whitman. Trent.

September 5. From Byron Sutherland, soldier. ст: Shively (2), 197.

September 11. From Louisa Van Velsor Whitman. Trent.

September 11. From Thomas Jefferson Whitman. ст: Berthold and Price, 11516.
September 20. From Abraham Simpson. pT: F. DeWolfe Miller, ed., Drum-Taps (1959), xlviii.

September 21. From Louisa Van Velsor Whitman. Trent.

September 27. From Louisa Van Velsor Whitman. Trent.

September 29. From Thomas Jefferson Whitman. ст: Berthold and Price, 11617.

October 17. From Ellen M. O'Connor.

October 19. From William D. O'Connor. Ст: Traubel, 1:83-85.

October 22. From Anson Ryder Jr. ст: Shively (2), 176.

October 25. From Margaret Stillwell. Berg. ст: Shively (2), 192-93.

November 1. From Ellen M. O'Connor.

November 11. From Benton H. Wilson, soldier. Ст: Shively (2), 213-14.

November 14. From Louisa Van Velsor Whitman. Trent. ст: Gohdes and Silver, 192.

November 25. From Louisa Van Velsor Whitman. Trent.

December 3. From Louisa Van Velsor Whitman. Trent.

December 10. From Louisa Van Velsor Whitman. Trent.

December 25. From Louisa Van Velsor Whitman. Trent.

December 28. From Margaret Stillwell. Berg. ст: Shively (2), 193-94.

\section{6}

February 20. From Jesse Mullery. LC.

February 21. From Charles J. Woodbury. G.W. Allen:Duke. ст: Barrus, 36-38.

March 27(?). From Louisa Van Velsor Whitman. Trent.

April 6. From Charlotte St. Clair, mother of a soldier.

April. From Charles L. Heyde. Trent. ст: Gohdes and Silver, 222-23.

May 31. From Louisa Van Velsor Whitman. Trent.

June 7. From Louisa Van Velor Whitman. Trent.

October 10. From Louisa Van Velsor Whitman. Trent.

November 12. From Bayard Taylor. ст: Traubel, 2:148-49. 
November 16. From Horace Wentworth. Yale.

November 26. From Alexander Gardner. ст: Traubel, 3:346.

November 27. From Horace Wentworth.

December 1(?). From Charles L. Heyde. Trent. ст: Gohdes and Silver, 223-24.

December 2. From Bayard Taylor. ст: Traubel, 2:153.

December 5. From Hugh B. Thomson, soldier.

December 9. From Benton H. Wilson, soldier. ст: Shively (2), 214.

December 13. From Hugh B. Thomson. Facsimile: Shively (2), 202, 203.

December 16. From Benton H. Wilson. Ст: Shively (2), 214-15.

December 17. From Horace Wentworth. Yale.

December 21. From Thomas Jefferson Whitman. ст: Berthold and Price, 11819.

\section{7}

January 1. From John T. Trowbridge.

January 4. From Senator George F. Edmunds.

January 17. From Louisa Van Velsor Whitman. Trent.

January 17. From Henry Wilson, father of soldier.

January 26. From Louisa Van Velsor Whitman. Trent.

January 27. From Benton H. Wilson. ст: Shively (2), 215-16.

February 3. From Benton H. Wilson. ст: Shively (2), 216-17.

February 8. From Charles Warren Stoddard.

February 19. From Louisa Van Velsor Whitman. Trent.

February 21. From Louisa Van Velsor Whitman. Trent.

February 24. From Anson Ryder Jr. ст: Shively (2), 177.

February 27. From Louisa Van Velsor Whitman. Trent.

March 15. From Louisa Van Velsor Whitman. Trent.

March 21. From Louisa Van Velsor Whitman. Trent.

[March 25]. From Abby H. Price. Yale.
March 28. From Louisa Van Velsor Whitman. Trent.

March. From Charles L. Heyde. Trent.

April 7. From Benton H. Wilson. ст: Shively (2), 217-18.

April 8. From James Curphey(?). LC.

April 13. From Louisa Van Velsor Whitman. Trent.

April 21. From Benton H. Wilson. ст: Shively (2), 219.

April 27. From Louisa Van Velsor Whitman. Trent.

May 2. From Louisa Van Velsor Whitman. Trent.

May 2. From H. J. Ramsdell, Washington clerk.

May 9. From William D. O'Connor. ст: Traubel, 3:521-22.

May 10. From Abraham Simpson.

May 19. From Charles F. Wingate, an admirer.

May 23. From Thomas Jefferson Whitman. ст: Berthold and Price, 119-22.

May 24. From Hiram Sholes. ст: Shively (2), 178-79.

May 31. From Abraham Simpson.

June 8. From Hiram Sholes.

June 20. From Louisa Van Velsor Whitman. Trent.

June 21. From Judge Milton Kelly. National Archives.

June. From Charles L. Heyde. Trent.

July 3. From Abraham Simpson.

July 17 . From H. J. Ramsdell.

July 20. From John T. Trowbridge. ст: Traubel, 3:506-7.

July 22. From H. J. Ramsdell.

August 1. From Louisa Van Velsor Whitman. Trent.

August 2. From Thomas Jefferson Whitman. Ст: Berthold and Price, 122-23.

August 8. From W. C. Church.

August 9. From William Livingston Alden. Ст: Traubel, 3:259.

August 9. From Judge Milton Kelly. Yale. ст: Shively (2), 152.

August 11. From Martha Whitman. Ohio Wesleyan. ст: Waldron, 42-43.

August 13. From F. P. Church.

August 14. From Scott \& Williams, printers.

August 19. From William Livingston Alden. 
August 26. From George W. Perrigo, a book order.

September 4. From F. P. Church.

September 10. From Moncure D. Conway. ст: Traubel, 3:267-68.

September 15. From Benton H. Wilson. CT: Shively (2), 219-20.

[September (?)] 29. From Alfred Pratt. ст: Shively (2), 168 (misdated August).

October 3. From Henry Clapp Jr. ст: Traubel, 1:267.

October 7. From Louisa Van Velsor Whitman. Trent.

October 12. From Moncure D. Conway. ст: Traubel, 3:296-98.

October 15. From F. P. Church.

October 20. From Louisa Van Velsor Whitman. Trent.

October 21. From F. P. Church.

October 22. From Louisa Van Velsor Whitman. Trent.

October 26. From Benjamin Russell Jr. Ст: Shively (2), 173-74 (misdated 1865).

October 30. From Louisa Van Velsor Whitman. Trent.

November 7. From Moncure D. Conway. ст: Traubel, 3:322.

November 13(?). From Louisa Van Velsor Whitman. Trent.

November 17. From William Michael Rossetti. ст: Traubel, 3:299-301; Peattie, 183-84 (misdated November 15).

November 18. From William Livingston Alden. ст: Traubel, 2:211.

November 19. From Louisa Van Velsor Whitman. Trent.

December 1-15(?). From Louisa Van Velsor Whitman. Trent.

December 6. From George S. McWatters.

December 8. From William Michael Rossetti. ст: Traubel, 3 :303-6; Peattie, 184-87.

December 15. From Louisa Van Velsor Whitman. Trent.

December 16. From William Michael Rossetti. ст: Traubel, 3:306-8; Peattie, 187-88.

December 23. From Dr. F. B. Gillette, an admirer. ст: Traubel, 3:465-66.

December 28. From George Routledge \& Sons. ст: Traubel, 1:263.
January 9. From A. Bronson Alcott. CT: Traubel, 3:243-44.

January 20. From William H. Millis Jr. Yale. ст: Shively (2), 159-60.

February 1. From Moncure D. Conway. Ст: Traubel, 2:284.

February 5. From John Camden Hotten. ст: Traubel, 2:285-86.

[February 12]. From Louisa Van Velsor Whitman. Trent.

[February] 17. From Louisa Van Velsor Whitman. Trent.

February 24. From Benton H. Wilson. ст: Shively (2), 220.

[February 25?]. From Louisa Van Velsor Whitman. Trent. ст: Gohdes and Silver, 192-94.

February 25. From A. Simpson \& Company.

[March 3]. From Louisa Van Velsor Whitman. Trent.

[March 6]. From Louisa Van Velsor Whitman. Trent.

March 11. From Louisa Van Velsor Whitman. Trent.

[March 13]. From Louisa Van Velsor Whitman. Trent. ст: Gohdes and Silver, 194-95.

March [24]. From Louisa Van Velsor Whitman. Trent.

[March 24]. From John M. Binckley.

March 25. From W. C. Church.

March 30. From Henry Wilson, father of an ex-soldier.

March 31. From Louisa Van Velsor Whitman. Trent. ст: Gohdes and Silver, 200-1 (dated 1869?).

April 7. From Louisa Van Velsor Whitman. Trent.

April 8. From John Camden Hotten. Yale. ст: Traubel, 4:308.

April 12. From William Michael Rossetti. Ст: Traubel, 2:123-24; Peattie, 194-95.

April 28. From A. Bronson Alcott. ст: Traubel, 3:245.

May 2. From F. P. Church.

May 3. From Benton H. Wilson. ст: Shively (2), 221.

May 5. From Louisa Van Velsor Whitman. Trent.

May 9. From Moncure D. Conway. Location unknown. ст: Traubel, 4:10. 
[May 14]. From Louisa Van Velsor Whitman. Trent. ст: Gohdes and Silver, 195-96.

May 15. From F. P. Church.

May 16. From Sheldon \& Company (for The Galaxy).

June 17. From Charles Hine, artist.

June 25. From Louisa Van Velsor Whitman. Trent. ст: Gohdes and Silver, 196-98.

July 1. From Louisa Van Velsor Whitman. Trent.

July 8. From Louisa Van Velsor Whitman. Trent.

July 12. From Thomas Jefferson Whitman. Ст: Berthold and Price, 125-27.

[July 15]. From Louisa Van Velsor Whitman. Trent.

[August 19]. From Louisa Van Velsor Whitman. Trent. ст: Gohdes and Silver, 198-99.

September 12. From Byron Sutherland. CT: Shively (2), 198.

September 16. From William D. O'Connor. Ст: Traubel, 2:431-32.

September 18. From Peter Doyle. Barrett. ст: Corr., 2:46n; Shively (1), 104-6.

September 23. From Peter Doyle. Typescript in Lion. Ст: Shively (1), 106.

September 24. From William F. Channing.

[September 25]. From John Swinton. ст: Traubel, 2:339-40.

September 27. From Peter Doyle. Morgan. Ст: Shively (1), 106-7.

October 1. From Peter Doyle. ms: Barrett. CT: Shively (1), 107.

October 5. From Henry Hurt.

October 5. From Peter Doyle. Lion. ст: Shively (1), 107-8.

October 6. From Benton H. Wilson. ст: Shively (2), 221.

October 8. From Byron Sutherland. ст: Shively (2), 199.

October 9. From Peter Doyle. Lion: NYPL. Ст: Shively (1), 108-9.

October 9. From William D. O'Connor.

October 14. From Peter Doyle. Barrett. CT: Shively (1), 109.

October 16. From Mrs. Henry Reynolds, mother of Walter Whitman Reynolds.

October 31. From Will W. Wallace, exsoldier. ст: Shively (2), 212-13.
November 2. From Louisa Van Velsor Whitman. Trent.

[November 4]. From Louisa Van Velsor Whitman. Trent.

November 8. From Alfred E. Pratt. Yale. CT: Shively (2), 169.

[After November 10]. From Louisa Van Veslor Whitman. Formerly in Hanley Collection.

[November 11]. From Louisa Van Velsor Whitman. Trent.

November 13. From Hannah Heyde. Trent.

November 17. From Alfred Wise, a jeweler's son. Yale.

[November 18]. From Louisa Van Velsor Whitman. Trent.

November 23. From William D. O'Connor. ст: Traubel, 3:504-5.

November 25. From Louisa Van Velsor Whitman. Trent.

[December 1]. From Louisa Van Velsor Whitman. Trent.

[December 5]. From Louisa Van Velsor Whitman. Trent.

December 5. From James T. Fields. Location unknown. ст: Traubel, 2:22.

[December 10]. From Louisa Van Velsor Whitman. Trent.

[December 14?]. From Louisa Van Velsor Whitman. Trent.

December 14. From James T. Fields. Location unknown. ст: Traubel, 2:211.

[December?] 16. From Louisa Van Velsor Whitman. Yale.

December 27. From Benton H. Wilson. Ст: Shively (2), 221-22.

December 28. From Louisa Van Velsor Whitman. Trent.

\section{9}

January 5. From John Morley. cr: Traubel, 1:216.

January 11. From John Flood Jr. Yale. CT: Shively (2), 143.

January 12. From Louisa Van Velsor Whitman. Trent.

January 19. From Louisa Van Velsor Whitman. Trent.

January 21. From Thomas Jefferson Whitman. ст: Berthold and Price, 13436. 
January 21. From Julius Sing(?), an admirer. LC.

January 24. From Benton H. Wilson. ст: Shively (2), 222-23.

[February 4]. From Louisa Van Velsor Whitman. Trent.

February 14. From Gabriel Sarrazin. Lion.

[February] 17. From Louisa Van Velsor Whitman. Trent.

[February 18?]. From Louisa Van Velsor Whitman. Trent.

[February]. From Louisa Van Velsor Whitman. Trent. ст: Gohdes and Silver, 199-200.

March 2. From Charles Warren Stoddard. ст: Traubel, 4:267-68.

March 4. From Louisa Van Velsor Whitman. Trent.

March 4. From W. C. Church.

[March 15]. From Louisa Van Velsor Whitman. Trent.

March 25. From Thomas Jefferson Whitman. Ст: Berthold and Price, 136-39.

[March] 31. From Louisa Van Velsor Whitman. Trent. ст: Gohdes and Silver, 200-1.

April 5. From Thomas Jefferson Whitman. Ст: Berthold and Price, 140-41.

April 7. From Louisa Van Velsor Whitman. Trent.

[April] 14. From Louisa Van Velsor Whitman. Trent.

[April 20?]. From Louisa Van Velsor Whitman. Trent.

June 23. From Louisa Van Velsor Whitman. Trent.

June 30. From Louisa Van Velsor Whitman. Trent.

[Summer]. From Louisa Van Velsor Whitman. Trent.

July 14. From Louisa Van Velsor Whitman. Trent.

July 18. From Benton H. Wilson. ст: Shively (2), 223.

July 22. From Hugh B. Thom[p]son, Yale. ст: Shively (2), 203.

August 10. From Dr. William A. Hawley. Syracuse. СT: Traubel, 4:365.

August 31. From Meredith R. Brookfield, an admirer. LC.

September 23. From Louisa Van Velsor Whitman. Trent.
[September 30]. From Louisa Van Velsor Whitman. Trent.

October [19]. From Louisa Van Velsor Whitman. Trent.

November 10. From Louisa Van Velsor Whitman. Trent.

November 16. From Walter Whitman Reynolds.

December 7. From Louisa Van Velsor Whitman. Trent.

December 19. From Benton H. Wilson. ст: Shively (2), 223-24.

[December 22?]. From Louisa Van Velsor Whitman. Trent.

December 23. From Thomas Dixon.

\section{0}

January 9. From William Michael Rossetti. Ст: Traubel, 1:379-81; Peattie, 247-48.

January 14. From Alfred and John B. Pratt. CT: Shively (2), 170-71.

January 19. From Louisa Van Velsor Whitman. Trent.

February 8. From Helen A. Horner, ecstatic admirer.

February 9. From Walter Whitman Reynolds.

February 25. From Edward C. Stewart. CT: Shively (1), 110-12.

February 27. From Martha Whitman. Ohio Wesleyan. ст: Waldron, 68-69.

March 1. From Mannahatta Whitman.

March 1. From Martha Whitman. Ohio Wesleyan. ст: Waldron, 69-70.

March 15. From John B. and N. M. Pratt. Yale. ст: Shively (2), 171-72.

March 22. From Dr. E. Warner, doctor who attended WW's brother Jesse. ст: Traubel, 1:294.

March [23?]. From Louisa Van Velsor Whitman. Trent.

[March 24]. From Louisa Van Velsor Whitman. Trent. ст: Gohdes and Silver, 201-2.

[March 28]. From Louisa Van Velsor Whitman. Trent. ст: Gohdes and Silver, 202-3.

March 30. From Byron Sutherland. Ст: Shively (2), 199-200.

March 30. From Martha Whitman. Ohio Wesleyan. ст: Waldron, 70-71. 
March. From Jessie Louisa Whitman. LC. April 2. From Charles Warren Stoddard. CT: Traubel, 3:444-45.

April 5. From Louisa Van Velsor Whitman. Trent.

April 8. From Byron Sutherland. ст: Shively (2), 200-1.

April 9. From Thomas Dixon.

[April 13]. From Louisa Van Velsor Whitman. Trent.

April 13. From Bret Harte (for The Overland Monthly). CT: Traubel, 1:28.

April 26. From Walter Whitman Reynolds.

May 15. From Thomas Bainbridge, exsoldier. Yale. ст: Shively (2), 107-8.

May 15. From Benton H. Wilson. CT: Shively (2), 224.

May 28. From Thomas Dixon.

May. From Stephen K. Winant, exsoldier. Yale. cT: Shively (2), 225-26.

June 1. From Louisa Van Velsor Whitman. Trent.

June 1. From Walter Whitman Reynolds.

June 8(?). From Louisa Van Velsor Whitman. Trent.

June 13. From Charles L. Heyde. Trent. ст: Gohdes and Silver, 226-28.

June 14. From Mrs. Nellie Eyster, an admirer. ст: Traubel, 1:34-35.

June 22. From Louisa Van Velsor Whitman. Trent.

June 29. From Louisa Van Velsor Whitman. Trent.

[June?]. From Louisa Van Velsor Whitman. Trent.

[July 12]. From Louisa Van Velsor Whitman. Trent. ст: Gohdes and Silver, 203.

July 20. From Louisa Van Velsor Whitman. Trent.

October 19. From John Swinton. ст: Traubel, 2:487-88.

November 20. From Ellen M. O'Connor. Ст: The Long Islander, May 25, 1967.

December 19. From R. M. Bucke. ст: Traubel, 2:6-7; Lozynsky, 1.

December 22. From Louisa Van Velsor Whitman. Trent.
February 5. From Thomas M. Woodworth, ex-soldier. ст: Shively (2), 22829.

February 8. From Louisa Van Velsor Whitman. Trent.

February 9. From John M. Rogers, a Brooklyn driver. CT: Shively (1), 126.

[February] 14. From Louisa Van Velsor Whitman. Trent.

February 27. From John M. Rogers. ст: Shively (1), 127.

April 6. From John M. Rogers. ст: Shively (1), 127.

April 10. From F. P. Church.

April 10. From John M. Rogers. CT: Shively (1), 128.

[April 16?]. From Louisa Van Velsor Whitman. Trent.

April 23. From Cyril Flower.

May 16. From Thomas Russell(?).

June 1. From John M. Rogers. cr: Shively (1), 128.

[June 13?]. From Louisa Van Velsor Whitman. Trent.

July 9. From William Michael Rossetti. CT: Traubel, 1:132-33; Peattie, 274-75.

July 12. From Alfred Tennyson. Location unknown. Ст: Donaldson, 223-24; Corr., 2:125-26.

July 16. From Cyril Flower. ст: Traubel, 2:373.

July 23. From Edward Dowden. ст: Traubel, 1:133-35.

July 27. From Thomas Dixon.

August 1. From George Peyton, Charles E. Burd, and James B. Young, for the American Institute. CT: Traubel, 1:326.

August 4. From Mrs. Charles Hine, wife of the artist. Ст: Traubel, 3:330-31 (dated August 6).

August 17. From William Black, autograph request.

August 22. From Louisa Van Velsor Whitman. Trent.

August 23. From F. S. Ellis. cT: Traubel, 2:447-48.

August 24. From F. S. Ellis. ст: Traubel, $2: 448$.

September 3. From Anne Gilchrist. LC. ст: Corr., 2:134-38; Harned, 58-64.

September 5. From Edward Dowden. ст: Traubel, 1:224-25. 
September 6. From George Washington and Louisa Orr Whitman. Hanley. ст: Loving, 161.

September 10. From Richard J. Hinton. Mrs. Doris Neale.

September 11. From John W. Chambers, for the American Institute. CT: Traubel, $4: 484$.

September 13. From Moncure D. Conway. Ст: Traubel, 3:111-12.

September 14. From Philip Hale, youthful admirer. ст: Traubel, 3:533.

[September 30]. From Louisa Van Velsor Whitman. Trent.

September 30. From Joaquin Miller. ст: Traubel, 1:107.

[September?]. From Louisa Van Velsor Whitman. Trent.

October 3-4. From John Burroughs. ст: Traubel, 1:89-90.

October 7. From John Addington Symonds. ст: Schueller and Peters, 2:16667.

October 8. From William Michael Rossetti. CT: Traubel, 3:376-77; Peattie, 280-81.

October 10. From Louisa Van Velsor Whitman. Trent. ст: Gohdes and Silver, 203-4.

October 15. From Edward Dowden. ст: Traubel, 3:41-42.

October 19. From Rudolf Schmidt. LC.

October 20. From Cyril Flower. cT: Traubel, 4:461-63.

October 23. From Anne Gilchrist. LC. ст: Harned, 65-66.

October 30. From John Burroughs. Syracuse.

November 3. From Roden Noel. ст: Traubel, 1:425-26.

[November 27]. From Anne Gilchrist. LC. ст: Corr., 2:141-43.

1871. From the Census Office, Department of the Interior. Trent.

\section{2}

[January 1]. From Louisa Van Velsor Whitman. Yale.

January 5. From Rudolf Schmidt. Syracuse. ст: Traubel, 4:103.

[January 10?]. From Louisa Van Velsor Whitman. Trent.
January 24. From Anne Gilchrist. LC. ст: Harned, 72-74.

January 31. From Matt H. Carpenter. Syracuse. ст: Traubel, 4:120.

February 5. From Rudolf Schmidt.

February 7. From John Addington Symonds. ст: Traubel, 1:74-76; Schueller and Peters, 2:201-3.

February 18 (not sent until February 14, 1876). From Bram Stoker. Syracuse. Ст: Traubel, 4:181-85.

February 21. From H. Buxton Forman. CT: Traubel, 2:265-66.

February 25. From John Addington Symonds. ст: Traubel, 1:387-88; Schueller and Peters, 2:205.

February 27. From Rudolf Schmidt.

March 31. From William Michael Rossetti. Ст: Traubel, 3:141-45; Peattie, 284-86.

April 8. From Pedelia(?) Bates. LC.

April 12. From Anne Gilchrist. LC. CT: Harned, 76-78.

April 25. From Rudolf Schmidt. cr: Traubel, 1:274-75.

May 13. From Walter Whitman Reynolds.

June 3. From Anne Gilchrist. LC. Ст: Harned, 79-81.

June 17. From Emil Arctander, acting vice-consul for Denmark.

June 20. From Emil Arctander.

July 14. From Anne Gilchrist. LC. CT: Harned, 82-84.

August 9. From S. W. Green, printer.

September 3. From Edward Dowden. ст: Traubel, 2:80-81.

September 26. From Grace B. Haight. LC.

October 28. From Martha Whitman. Ohio Wesleyan. ст: Waldron, 83-85.

November 10. From Thomas Jefferson Whitman. ст: Berthold and Price, 14647.

November 12. From Anne Gilchrist. LC. CT: Harned, 85.

[December 3]. From Louisa Van Velsor Whitman. LC.

December 26. From John M. Rogers. Ст: Shively (1), 128-29.

[1872?]. From Fred Vaughan. Ст: Shively (1), 48-49. 


\section{3}

January 12. From John Burroughs. ст: Traubel, 3:281-82.

January 15. From R. W. Waldo (ordering a book).

[About January 20]. From Louisa Van Velsor Whitman.

[January] 30. From Louisa Van Velsor Whitman. Trent.

January 31. From Anne Gilchrist. LC. CT: Harned, 86-87.

February 7. From Thomas Jefferson Whitman. ст: Berthold and Price, 155.

February 11. From Thomas Jefferson Whitman. ст: Berthold and Price, 15657.

[February 12?]. From Louisa Van Velsor Whitman. Trent.

February 24. From Mannahatta Whitman.

February 27. From Louisa Van Velsor Whitman. Trent.

[February?]. From Louisa Van Velsor Whitman. Trent.

[March 1]. From Louisa Van Velsor Whitman. Trent.

March 4. From Louisa Van Velsor Whitman. Trent.

March 4. From Hannah Heyde. LC.

[March 10?]. From Louisa Van Velsor Whitman. Trent.

March 10. From George H. Williams. Lion. ст: Traubel, 3:475-76.

March 16. From Thomas Jefferson Whitman. Yale. ст: Berthold and Price, 16164.

March 19. From William F. Channing.

March 21. From Louisa Van Velsor Whitman. Trent.

[March 23?]. From Louisa Van Velsor Whitman. Trent.

March 28. From Sampson, Low \& Company.

March 30. From Thomas Jefferson Whitman. ст: Berthold and Price, 165-66.

[March]. From Louisa Van Velsor Whitman. Trent.

[March?] Saturday. From Louisa Van Velsor Whitman. Trent.

[April 2?]. From Louisa Van Velsor Whitman. Trent.

[April 3?]. From Louisa Van Velsor Whitman. Trent.
April 4. From Rudolf Schmidt.

[April 5?]. From Louisa Van Velsor Whitman. Trent.

[April 8]. From Louisa Van Velsor Whitman. Trent.

April 11. From John Burroughs.

[April 12]. From Louisa Van Velsor Whitman. Trent.

April 12. From Edward Dowden. cr: Traubel, 1:441-43.

[April 20?]. From Louisa Van Velsor Whitman. Trent.

[April 21]. From Louisa Van Velsor Whitman. Trent.

[May 1]. From Louisa Van Velsor Whitman. Trent.

[May 9?]. From Louisa Van Velsor Whitman. Trent.

May 9. From Thomas Jefferson Whitman. ст: Berthold and Price, 169.

[May 12?]. From Louisa Orr Whitman. LC.

[May 12?]. From Louisa Van Velsor Whitman. Trent.

May 14. From John Burroughs. Syracuse. Ст: Traubel, 4:304-5.

[May 15]. From Louisa Van Velsor and Louisa Orr Whitman. Hanley.

[May 17?]. From Louisa Van Velsor Whitman. Trent.

[About May 20]. From Louisa Van Velsor Whitman. Trent.

May 20. From Sarah Avery. Lc.

May 20. From Anne Gilchrist. LC. CT: Harned, 88-90.

June 2. From John Burroughs.

[June 5?]. From Louisa Orr Whitman. LC.

June 7. From Hannah Heyde. LC. ст: Life in Letters: American Autograph Fournal, 2(1939):103-4.

August 12. From Anne Gilchrist. LC. ст: Harned, 91-93.

August 17. From Hannah Heyde. Lc.

September 4. From Anne Gilchrist. LC. ст: Harned, 96-97.

November 1. From Henry M. Alden. Barrett.

November 3. From Anne Gilchrist. LC. CT: Harned, 98-101.

November 28. From G. A. White, WW's landlord.

December 1. From Henry M. Alden.

December 8-18. From Anne Gilchrist. Lc. ст: Harned, 102-4. 
December 9. From William Stansberry. Trent.

December 11. From Minnie Vịncent, autograph request.

December 12. From Edmund W. Gosse. ст: Traubel, 1:245-46; Victorian Studies, 1(1957):181.

\section{4}

January 2. From Rudolf Schmidt. CT: Traubel, 3:361.

January 12. From Walt Whitman Storms. January 19. From David G. Croly. Ст: Traubel, 3:560-61.

February 16. From William H. Millis Jr. Berg. CT: Shively (2), 160.

[February 25]. From Anne Gilchrist. LC. ст: Harned, 105-7 (dated February 26).

February 28. From Rudolf Schmidt.

March 6. From Leon P. Luckey, President Grant's secretary.

March 9. From Anne Gilchrist. LC. ст: Harned, 108.

March 9. From Walt Whitman Storms.

March 20. From J. C. Mann. Lion.

March 20. From Rudolf Schmidt. Ст: Traubel, 4:356-58.

April 2. From J. C. Mann. Lion.

April 4. From Rudolf Schmidt.

May 12. From William Stansberry. Trent.

May 14. From Anne Gilchrist. LC. ст: Harned, 109-11.

May 17. From John Burroughs. Cт: Traubel, 2:43-44.

May 26. From Thomas A. Wilson (a real estate transaction).

June 1. From Walter Godey. LC.

June 21. From William H. Taylor, a former driver.

June 23. From John Swinton. ст: Traubel, 1:24-25 (misdated January 23, 1884).

June 25. From Rudolf Schmidt. Syracuse. CT: Traubel, 4:464-65 (misdated January 26$)$.

June 28. From William Stansberry. Trent.

June 30. From George H. Williams. Yale. ст: Traubel, 3:476.

July 4-6. From Anne Gilchrist. LC. Ст: Harned, 112-14.
July 6. From George H. Williams. Copy in National Archives.

July 8. From Alfred Tennyson. Location unknown. Ст: Donaldson, 227-28; Corr., 2:307-8.

July 12. From Edward Carpenter. Ст: Traubel, 1:158-61.

July 15. From Mrs. Jane Stansberry. Trent.

July 16. From Colonel John R. Johnston. Fragment in Barrett.

July 28. From Rudolf Schmidt.

July 29. From Mrs. Isabella A. White, WW's landlady.

August 11. From Fred Vaughan. ст: Shively (1), 48-49.

August 13. From John Newton Johnson.

August 24. From J. P. Young \& Company.

September 2. From Thomas Gibbons, autograph request.

September 3-4. From Anne Gilchrist. LC. CT: Harned, 115-18.

September 8. From Thomas Dixon.

September 13. From John Newton Johnson.

October 6. From Mrs. Isabella A. White.

October 7. From John Newton Johnson.

[November 6]. From Joaquin Miller. ст: Traubel, 1:44-45.

November 7. From John Newton Johnson.

November 16. From Fred Vaughan. ст: Shively (1), 49-50.

December 9. From Anne Gilchrist. LC. ст: Harned, 119-20.

December 10. From Mrs. Maria Smith, mother of ex-soldier.

December 15. From Joseph B. Marvin. LC.

December 22. From Whitelaw Reid. ст: Traubel, 1:463-64.

December 28. From Rudolf Schmidt.

December 30-January 1, 1875. From Anne Gilchrist. LC. ст: Harned, 121-22.

\section{5}

February 1. From Mrs. Maria Smith.

February 8. From John Newton Johnson.

February 11. From Ruben Farwell. Trent. ст: Shively (2), 141.

February 12. From James Redfield. 
February 16. From Elizabeth Ford, English admirer.

February 19. From John Newton Johnson. PT: Barrus, 92-93.

February 21-25. From Anne Gilchrist. LC. Cт: Harned, 123-25.

February 28. From William H. Millis Jr. Berg. Ст: Shively, (2), 160 (dated February 26).

March 1. From Manvill Wintersteen, exsoldier. Berg. ст: Shively (2), 227.

March 5. From Ruben Farwell. Trent. CT: Shively (2), 141-42.

March 10. From Manvill Wintersteen. Berg. CT: Shively (2), 227-28.

March 12. From Bethuel Smith. CT: Shively (2), 186 (misdated 1874).

March 14. From Mrs. Maria Smith.

March 16. From Charles P. Somerby.

April 3. From John Newton Johnson.

April 4. From William H. Millis Jr. Berg. CT: Shively (2), 161.

April 14. From William Michael Rossetti. ст: Peattie, 322-23.

April 15. From Thomas Dixon.

April [18?]). From John M. Rogers. Feinberg:LC. CT: Shively (1), 129.

April 20. From Walt Whitman Storms.

April 25. From John M. Rogers. ст: Shively (1), 129-30.

April 26. From John Newton Johnson.

April 30. From John T, Trowbridge. ст: Traubel, 2:224 (misdated April 3).

May 5. From Charles P. Somerby.

May 10. From John Newton Johnson.

May 18. From Anne Gilchrist. LC. ст: Harned, 126-28.

May 19. From William J. Linton. ст: Traubel, 1:12-13.

May 27. From Joaquin Miller. ст: Traubel, 1:57.

May 31. From Will Williams (for The Pictorial World).

June 8. From Edmund Clarence Stedman. Location unknown. СT: Donaldson, 213-14.

June 13: From John Addington Symonds. Ст: Traubel, 1:203-4; Schueller and Peters, $2: 374-75$.

June 14. From John M. Rogers. ст: Shively (1), 130.

June 23. From Benton H. Wilson. Berg. CT: Shively (2), 225.
July 5. From Thomas Jefferson Whitman. СT: Berthold and Price, 171.

July 17. From Joseph C. Baldwin. Hanley: Texas.

July 17 . From Rudolf Schmidt.

[July 20]. From John Newton Johnson.

July 21. From William Stansberry. Trent.

July 22. From Frederic R. Marvin (a request to print one of WW's poems). LC.

July 24. From John M. Rogers. Ст: Shively (1), 130-31.

July 27. From John Burroughs.

August 8. From Manvill Wintersteen. Berg. ст: Shively (2), 228.

August 9. From Walt Whitman Storms.

[August?] 10. From John Newton Johnson.

August 11. From Alfred Tennyson. Location unknown. Text: Henkels, October 26. 1899. ст: Donaldson, 229-30 (dated August 16); Corr., 2:339.

August 16. From Ruben Farwell. Trent. CT: Shively (2), 142.

August 18. From Rudolf Schmidt. Location unknown. ст: Traubel, 4:336-38.

August 21. From William J. Linton. ст: Traubel, 2:201-2.

August 28. From Anne Gilchrist. LC. ст: Harned, 129-30.

September 5. From Joaquin Miller. ст: Traubel, 1:360-61.

September 25. From Charles P. Somerby. September 27. From William H. Millis Jr. Berg. ст: Shively (2), 161.

September 27. From Lord Houghton (Richard Monckton Milnes). ст: Traubel, 1:364 (dated September 29).

October 4. From Charles P. Somerby.

October 7. From Philip Hale. Lc.

November 3. From Lord Houghton. ст: Traubel, 2:310.

November 7. From Peter Doyle. ст: Shively (1), 114.

November 9. From John Newton Johnson.

November 13. From George D. Cole, Washington railroad conductor. Yale.

November 14. From George Washington Whitman. Yale.

November 16-30. From Anne Gilchrist. LC. ст: Harned, 133-36.

November 18. From Edwin Einstein. 
November 25. From Josie Morse, autograph request.

December 4. From Anne Gilchrist. LC. cT: Harned, 137-38.

December 12. From Richard J. Hinton. Livesey.

December 19. From Thomas Dixon.

December 23. From William Michael Rossetti. ст: Peattie, 332-33.

[December?] 27. From John Newton Johnson.

1875. From John Newton Johnson.

\section{6}

January 2. From Jeannette L. Gilder. LC. January 3. From Edward Carpenter. PT: Traubel, 3:414-18.

January 18. From Anne Gilchrist. LC. ст: Harned, 139-40.

January 26. From H. Buxton Forman. CT: Traubel, 2:266-67.

February 6. From Edward Dowden. ст: Traubel, 1:299-301.

[February 7]. From John Newton Johnson.

February 14. From Bram Stoker. CT: Traubel, 4:180-81.

February 16. From Thomas Dixon.

February 16. From Edward Dowden. ст: Traubel, 1:301-3.

February 17. From E. A. Ellsworth.

February 18. From Alfred Webb (ordering books).

February 25. From Anne Gilchrist. LC. CT: Harned, 141-42.

February 28. From William Michael Rossetti. CT: Peattie, 335-36.

February 29. From Kenningale Cook (ordering books).

March 11. From Anne Gilchrist. LC. СT: Harned, 143-44.

March 15. From Nancy M. Johnson (ordering a book).

March 16. From Edward Dowden. ст: Traubel, 1:122-23.

March 17. From E. Mell Boyle(?) (willing to advertise Leaves of Grass).

March 20. From Henry Abbey(?).

March 28. From John M. Rogers. ст: Shively (1), 131.

March 30. From Anne Gilchrist. LC. ст: Harned, 147-48.
March 30. From William Michael Rossetti.

March 30. From Therese C. Simpson and Elizabeth J. Scott-Moncrieff, admirers.

April 2. From Albert G. Knapp, an exsoldier. Berg. Ст: Shively (2), 54-55.

April 4. From William Michael Rossetti. ст: Peattie; 339-40.

April 7. From E. F. Strickland Jr. (autograph request).

April 8. From Edward Carpenter.

April 9. From Charles Hine (ordering a book).

April 14. From B. G. Morrison (ordering books).

April 15. From Dr. Ferdinand Seeger (payment for a book).

April 16. From Joaquin Miller. ст: Traubel, 2:139-40.

April 18. From Robert Buchanan.

April 18. From Rudolf Schmidt.

April 18. From Dr. Ferdinand Seeger (ordering a book).

April 19. From Charles P. Somerby, a bookseller.

April 21. From Anne Gilchrist. LC. ст: Harned, 149-51.

April 22. From J. M. Green (requesting WW act as poet at Dickinson College).

April 23. From John Quincy Adams Ward. ст: Traubel, 2:278.

April 24. From Moncure D. Conway. CT: Traubel, 1:346-47.

April 24. From John Swinton (ordering books). ст: $A L, 39$ (1968): 549.

April 25. From the Rev. A. P. Putnam (ordering books).

April 26. From D. Jardine (ordering a book).

April 26. From John T. Trowbridge (payment for a book).

April 27. From A. J. Davis (ordering books).

April 28. From Robert Buchanan.

May 1. From Susan Stafford. ст: Shively (1), 150.

May 2. From Charles W. Eldridge. ст: Traubel, 3:483.

May 3. From Laura Curtis Bullard. ст: Traubel, 3:555-56.

May 3. From S. W. Green, a New York printer.

May 7. From John Newton Johnson. 
May 10. From S. F. Michel (?), for the Chicago Tribune (returning a manuscript).

May 12. From Charles P. Somerby.

May 18. From Anne Gilchrist. LC. Ст: Harned, 152-53.

May 24. From Miss R. M. Cox (ordering a book).

June 1. From John Quincy Adams Ward (payment for books).

June 3. From Edward Carpenter.

June 17. From Thomas Dixon.

July 5 . From John Newton Johnson.

July 10. From Whitelaw Reid. ст: Traubel, 2:212.

July 17. From John Newton Johnson.

July 24. From Rudolf Schmidt.

July 25. From John Hay. CT: Traubel, 1: 60 (dated July 22).

[August 5]. From John Newton Johnson.

August 21. From James Arnold (acknowledging receipt of books).

September 23. From Justin H. McCarthy Jr. (acknowledging receipt of a book).

September 28. From Charles P. Somerby.

October 4. From Edward Dowden. ст: Traubel, 2:90-91.

[October 6]. From John Newton Johnson.

November 12. From William Gardner Barton (autograph request).

November 17. From W. A. Stagg (autograph request).

[About December 20]. From John Newton Johnson.

December (?). From William Michael Rossetti.

1876(?). From George Fraser. LC.

\section{7}

January 1. From Eugene Benson, a painter. LC.

January 8. From Robert Buchanan. CT: Traubel, 1:2-3.

January 23. From John Addington Symonds. Ст: Traubel, 1:458-59; Schueller and Peters, 2:446-47.

January 25. From D. M. Zimmerman (requesting an article). LC.

January 31. From Susan Stafford.

February 1(?). From Thomas B. Freeman (acknowledging receipt of a book).
February 20. From John Newton Johnson.

March 1. From Edward Carpenter. Syracuse.

March 27. From John Newton Johnson.

March 27. From W. A. B. Jones (autograph request).

March 29(?). From Scribners, Armstrong Company. LC.

April 6. From Sarah E. Bowen (or Brown), a friend of Amelia Johnston. LC.

April 21-22. From Harry Stafford. ст: Shively (1), 150-51.

April 23. From Kenningale Cook. ст: Traubel, 2:219.

May 13. From Joseph C. Baldwin. ст: Shively (1), 132-34.

May 13. From Thomas B. and P. J. Freeman.

May 20. From John Newton Johnson.

May 21. From Harry Stafford. cr: Shively (1), 152 (dated May 1).

May 31. From Trübner \& Company. LC.

June 15-22. From William Michael Rossetti. CT: Traubel, 3:170-72; Peattie, 350-52 (including a copy of R. D. Adams' review of WW).

July 9. From Harry Stafford. ст: Shively (1), 152.

[July 12]. From St. Loe Strachey (sending verses).

July 12. From John Addington Symonds. CT: Traubel, 3:197; Schueller and Peters, 2:484-86.

July 21. From Harry Stafford. Ст: Shively (1), 153.

July 22. From Thomas Jefferson Whitman. Ст: Berthold and Price, 172-73.

August 2. From Kate A. Evans, a "rather gushing" admirer.

August 6. From Harry Stafford. ст: Shively (1), 153-54.

August 10. From John Burroughs. Ст: Traubel, 2:318-19.

August 11. From Joseph C. Baldwin. ст: Shively (1), 134.

August 14. From Harry Stafford. ст: Shively (1), 154.

August 17. From William Michael Rossetti.

September 1. From Walt Whitman Storms. 
September 11. From Joseph C. Baldwin. CT: Shively (1), 134.

September 16. From John Newton Johnson.

September 17. From Professor R. B. Anderson (requesting appointment).

September 17. From Edward Carpenter. Syracuse. ст: Traubel, 4:204-5.

September 20. From Edward Carpenter. Location unknown. ст: Traubel, 4:205.

September 21. From Joseph C. Baldwin. CT: Shively (1), 135.

September 24. From Annie Talman Smith, an admirer. ст: $A L, 39$ (1968):350n.

[September 24]. From John Swinton (introducing Annie Talman Smith). ст: $A L, 39$ (1968): 549.

September 25. From Harry Stafford. ст: Shively (1), 154.

September 29. From John Burroughs. Location unknown. PT: Cope's Tobacco Plant, 2 (1879): 319; $C W, 4: 192-93$.

October 3. From Claxton, Remsen, \& Haffelfinger, booksellers. LC.

October 4. From Harry Stafford. Ст: Shively (1), 154-55.

October 10. From Charles L. Heyde. Trent.

October 17. From Harry Stafford. ст: Shively (1), 155.

October 21. From Edward P. Cattell. ст: Shively (1), 156.

October 24. From Harry Stafford. cT: Shively (1), 156-57.

October 29. From Harry Stafford. CT: Shively (1), 157.

November 2. From Harry Stafford. CT: Shively (1), 157-58.

November 4. From R. M. Bucke. ст: Lozynsky, 2.

November 7. From Harry Stafford. cT: Shively (1), 158-59.

November 9. From Elmer E. Stafford. cT: Shively (1), 159.

November 13. From Harry Stafford. ст: Shively (1), 159.

[November 15]. From Edward D. Bellows (ordering books). LC.

November 17. From Harry Stafford. ст: Shively (1), 159-60.

November 21. From Harry Stafford. ст: Shively (1), 160.
November [26]. From Edward Cattell. ст: Shively (1), 160.

November 27. From Harry Stafford. ст: Shively (1), 161.

December 2. From John T. Trowbridge. CT: Traubel, 2:224-25.

December 17. From William Michael Rossetti.

December 18. From William H. Taylor (inviting WW for a visit). LC.

December 19. From Edward Carpenter. ст: Traubel, 1:189-190.

December 23. From Susan Stafford.

[December]. From Joaquin Miller. ст: Traubel, 3:225.

[1877(?)]. Friday. From Frederick Wedmore.

\section{8}

January 5. From Claxton, Remsen, \& Haffelfinger, booksellers. LC.

January 11. From Elmer E. Stafford. CT: Shively (1), 161-62.

January 18. From Elmer E. Stafford. CT: Shively (1), 162-63.

January 18. From Harry Stafford. CT: Shively (1), 162.

January 20. From Peter Doyle(?). LC. Ст: Shively (1), 114.

January 24. From Harry Stafford. Cт: Shively (1), 163.

January 26. From Susan Stafford.

January 29. From Harry Stafford. ст: Shively (1), 171 (dated 1892).

January 29. From W. L. Tiffany.

January 31. From C. B. Whitman (asking for genealogical data).

[January]. From George William Foote.

February 3. From John Burroughs. Lion. Ст: Traubel, 4:463-64.

[February 8]. From John Newton Johnson.

February 21. From John M. Rogers. ст: Shively (1), 131-32.

February 23. From James Matlack Scovel. LC.

February 25. From James Matlack Scovel. LC.

February 25. From John Burroughs. PT: Memoranda During the War, ed. Roy P. Basler (1962), 27-28.

February 28. From John Burroughs. 
March [14]. From John Newton Johnson. March 16. From Mary Van Nostrand. Trent. ст: Gohdes and Silver, 207-8.

March 26. From Harry Stafford. CT: Shively (1), 163-64.

March 27. From Charles A. Raymond(?). Barrett.

April 4. From Susan Stafford.

April 11. From members of Sarah Mead's family (announcing Mrs. Mead's death).

April 20. From George Parsons Lathrop. ст: Traubel, 2:315-16.

May 5. From John Newton Johnson.

May 5. From Sidney Lanier. Facsimile: Traubel, I, 208. ст: Conservator, 7 (October 1896): 122; Sidney Lanier: Centennial Edition, 10:40-41n.

May 13. From Edward Carpenter. Barrett. Ст: Traubel, 4:391-92.

June 5. From Harry Stafford. Ст: Shively (1), 164.

June 7. From Mrs. Walter Bownes, a relative. LC.

July 11. From John Burroughs.

July 17. From Whitelaw Reid. Mrs. Doris Neale.

July 27. From Harry Stafford. ст: Shively (1), 165.

August 3. From Benjamin Gurney(?) (acknowledging receipt of books for $\mathrm{Mr}$. Sarony). Hanley.

August 11. From Oscar Tottie (acknowledging receipt of books). LC.

[August 12]. From Beatrice Gilchrist. LC. ст: Harned, 156-58.

August 24. From Alfred Tennyson. Hanley. ст: Corr., 3:134-35.

August 26. From Harry Stafford. PT: Shively (1) 165-66.

September 3. From Anne Gilchrist. LC. CT: Harned, 159-60.

September. From Anne Gilchrist. LC. ст: Harned, 154-55 (dated 1877).

October 7. From George W. Bull (requesting autograph). LC. ст: C. J. Furness, Walt Whitman's Workshop (1928), 200.

October 25. From Anne Gilchrist. LC. CT: Harned, 161-62.

October 27. From Thomas Jefferson Whitman. ст: Berthold and Price, 17375.
November 13. From Anne Gilchrist. LC. CT: Harned, 163-65.

[December 14]. From John Newton Johnson.

December 29. From Susan Stafford. Ст: Shively (1), 166.

\section{9}

January 2. From Hannah Heyde. Lc.

January 5. From Anne Gilchrist. LC. CT: Harned, 166-68.

January 13. From John Burroughs.

January 13. From Harry Stafford. ст: Shively (1), 166-67.

January 14. From Anne Gilchrist. LC. CT: Harned, 169-70.

January 23. From Nancy Whitman(?).

January 27. From Anne Gilchrist. LC. CT: Harned, 171-72.

February 2. From Herbert Gilchrist. LC. ст: Harned, 173-74.

February 7. From James Matlack Scovel. Livezey. ст: WWR, 7 (1961):7.

February 16. From Beatrice Gilchrist. LC. cт: Harned, 175-76.

March 5. From William Harrison Riley, a friend of John Ruskin.

March 18. From Anne Gilchrist. LC. Ст: Harned, 177-78.

March 26. From Anne Gilchrist. LC. CT: Harned, 179-80.

April 2. From William Harrison Riley. April 4. From William Harrison Riley.

May 29. From D. Nicholson (for the New York Tribune). LC.

June 9. From Alfred Janson Bloor. LC.

June 20. From Anne Gilchrist. LC. Ст: Harned, 181-82.

July. From T. W. H. Rolleston. Manchester.

July 3. From Horace L. Traubel. Lc.

July 5. From the Office of the Recorder, Katonah, N. H. (ordering a book). Livezey.

July 16. From an unidentified correspondent (requesting autograph). Livezey.

July 17. From G. P. Putnam's Sons. LC.

August 2. From Anne Gilchrist. LC. ст: Harned, 183-85.

[August 9()]. From Sidney H. Morse.

August 13. From H. B. Wilson, Camden Postmaster. LC. 
August 24. From John Burroughs. ст: Traubel, 3:260-61.

August 25. From P. Armáchalain, "the Hindoo."

August 27. From William H. Kelly (ordering a book). LC.

September 3. From Charles T. Dillingham (probably ordering books). Fragment in Daybooks, 1:153.

September 29. From Richard Worthington. Yale.

October 1. From Richard Watson Gilder. Historical Society of Pennsylvania. ст: Donaldson, 215-16.

October 6-12. From Anne Gilchrist. Barrett. ст: Clara Barrus, Whitman and Burroughs-Comrades (1931), 147-48.

October. From Anne Gilchrist. Livezey. Ст: WWR, 7 (1961): 12.

November 9. From R. M. Bucke. ст: Lozynsky, 3.

November 25. From E. Steiger (probably ordering books). Fragment in Daybooks, 1:160, 166.

December 5. From Anne Gilchrist. LC. ст: Harned, 187-89.

December 29. From John Burroughs. Manchester. ст: Emerson Society Quarterly, 43, II Quarter (1966): 67-68.

[Before 1880] From A. B. Ashley (autograph request). Livezey.

[Before 1880] From J. L. Smith (autograph request). Livezey.

\section{0}

January 19. From R. M. Bucke. Manchester. Ст: Lozynsky, 4.

January 20. From James W. Thompson (ordering books).

January 25. From Anne Gilchrist. LC. ст: Harned, 190-92.

January 31. From Herbert J. Bathgate.

February 3. From R. M. Bucke. Manchester. ст: Lozynsky, 5.

February 6. From R. M. Bucke. Manchester. ст: Lozynsky, 6.

February 15. From William Mills (acknowledging receipt of a book and photograph).

March 9. From Herbert Gilchrist. Location unknown. PT: Barrus, 148-49.
March 18. From R. M. Bucke. Manchester. Ст: Lozynsky, 7.

March 23. From R. M. Bucke. Manchester. Ст: Lozynsky, 8.

March 25. From Robert G. Ingersoll. Manchester.

[March 28]. From Edward Carpenter. Manchester.

March 28. From Anne Gilchrist. Manchester.

April 3. From James Berry Bensel, a young admirer.

April 7. From Frederick LockerLampson. Location unknown. ст: Donaldson, 236-37.

[April or May]. From T. W. H. Rolleston. Manchester.

June 5. From Katie Macdonald (ordering a book). Manchester.

June 9. From Katie Macdonald (sending payment for book). Manchester.

June(?) 9. From William H. Taylor (requesting an article). Manchester.

June 10. From A. H. Whitaker, an admirer.

June 14. From Charles Warren Stoddard. Manchester.

June 15. From Anne Gilchrist. Whitman House, Camden.

June 15. From Frederick LockerLampson. Manchester.

June 18. From Mrs. Elisa S. Leggett. Manchester.

June 21. From James Matlack Scovel. Manchester.

June 22. From Harry Scovel. Manchester. June 24. From C. A. J. Hueckberny(?) (acknowledging receipt of WW's note). Manchester.

June 24. From Fred W. Rauch (concerning his travels in Germany). Manchester.

June 26. From John H. Johnston. Manchester.

June 27. From Norman McKenzie, a Canadian schoolboy. Manchester.

July 1. From Edward Carpenter. Manchester.

July 2. From Herbert J. Bathgate. Manchester.

July 3. From Frederick Locker-Lampson. Manchester. 
July 4. From Respegius Edward Lindell. Manchester.

July 4. From Louisa Orr Whitman. Manchester.

July 7. From Charles Warren Stoddard. Manchester.

July 9. From Robert Elliott, a young Canadian admirer. Manchester.

July 10. From Josiah Child (sending a draft from Trübner \& Co.). Manchester.

July 10(?). From Harry Stafford. Manchester.

July 16. From Susan(?) Stafford. Manchester.

July 17. From Elmer E. Stafford. Manchester.

[July 17]. From Harry Stafford. Manchester. ст: $W W Q R, 8$, no. 4 (Spring 1988): 43-44.

July 18. From Deborah Stafford Browning. Manchester.

July 19. From Mrs. Elisa S. Leggett. Manchester.

July 21. From Franklin B. Sanborn. Manchester.

July 22. From Louisa Orr Whitman. Manchester.

August 1. From John H. Ingram.

August 4. From Vivas(?) Tully (furnishing data about the Great Lakes). Manchester.

August 22. From Anne Gilchrist. LC. ст: Harned, 193-94.

September 8. From Jack Richardson. LC. Cт: L'Ane d'Or, 5 (1926); 44-45.

October 9. From Mrs. Elisa S. Leggett. LC. ст: Donaldson, 239-42.

October 15. From Frederick LockerLampson. Location unknown. ст: Donaldson, 237.

October 14. From William Davidge(?) (autograph request).

October 16. From T. W. H. Rolleston. ст: Traubel, 2:67-69.

October 20. From E. C. Cheever (ordering books).

October 25. From C. L. Ehrenfeld (ordering books).

October 25. From George Heard (ordering books).

October 27. From Frederic Almy (enclosing payment for books).
November 2. From John Burroughs. Hanley.

November 10. From Jno. S. Stott.

November 11. From W. Colin Mackenzie.

November 11. From T. W. H. Rolleston. LC. Ст: Frenz, 19-20.

November 20. From Helena de Kay Gilder. ст: Traubel, 2:118-19.

November 20. From David Hutcheson (ordering books).

November 22. From Titus M. Coan, The Century Club (ordering books). LC.

November 28. From Lizzie Westgate, fervid young admirer.

November 30. From Herbert Gilchrist. LC. CT: Harned, 195-96.

December 2. From A. Williams (ordering books).

December 6. From James Matlack Scovel.

December 10. From Leon Richeton (requesting photograph for an etching). Lion.

December 10. From Hannah L. Taylor (thanking the poet).

December 13. From Herbert Gilchrist.

December 29. From James Hearne, The Century Club (requesting a receipt). LC.

[1880?]. From Harry Stafford. LC. Ст: $W W Q R, 5$, no. 4 (Spring 1988): 40-41.

\section{1}

January 20. From W. S. Kennedy. ст: Traubel, 1:377-78.

January 21. From Charles Allen Thorndike Rice. Yale.

January 25. From the Camden \& Atlantic Railroad Company. LC.

January 29. From T. W. H. Rolleston. LC. Ст: Frenz, 21-24.

January 31. From Frederick LockerLampson. Historical Society of Pennsylvania.

February 10. From T. W. H. Rolleston. LC. Ст: Frenz, 25-27.

February 16. From Anne Gilchrist. Pennsylvania.

February 21. From Mrs. Mollie W. Carpenter, a young poet. LC.

February 22. From Henry Wadsworth Longfellow. Berg. ст: The Letters of Henry Wadsworth Longfellow, ed. Andrew Hilen (1966-82):6:692. 
February 25. From Elihu Vedder (ordering books).

February 28. From John Alcott (acknowledging receipt of books). LC.

March 14. From John Burroughs. Ст: Traubel, 1:43-44; Barrus, 199-200.

March(?). From T. W. H. Rolleston. LC. Ст: Frenz, 28-29 (dated February-May 1881).

April 4. From Harry Stafford. ст: Shively (1), 169.

April 15. From J. T. Cobb, a troubled admirer.

April 18. From Anne Gilchrist. LC. Ст: Harned, 197-99.

April 26. From John Boyle O'Reilly. Huntington.

May 12. From James R. Osgood. LC. PT: $C W, 8: 277$.

May 16. From Henry A. Beers. LC.

May 19. From Emma M. String, of the Pennington Seminary (inviting WW to a dedication).

May 22. From R. M. Bucke. ст: Lozynsky, 9.

May 23. From John Burroughs. Hanley.

May 23. From James R. Osgood. LC. PT: $C W, 8: 278$ (dated May 28).

[May(?)] 24. From Henry M. Alden (returning a poem). LC.

May 31. From James R. Osgood. LC. PT: $C W, 8: 278-79$.

June 3. From James R. Osgood. LC. PT: $C W, 8: 279-80$.

June 4. From T. W. H. Rolleston. LC. cT: Frenz, 30-34.

June 5. From Herbert Gilchrist. LC. CT: Harned, 200-2.

[June 6?] undated. From Louis G. Richardson (asking for information about WW's books). LC. See Daybooks, 243.

June 8. From Benjamin Ticknor. LC.

June 10. From James R. Osgood \& Company. LC.

June 17. From Anne Gilchrist. Pennsylvania.

June 21. From James R. Osgood \& Company. LC.

June 22. From Mrs. Elisa S. Leggett. New-York Historical Society. ст: Donaldson, 242-46.

June 25. From James R. Osgood \& Company. LC. PT: $C W, 8: 282$.
July 1. From Edward Carpenter.

July 11. From T. W. H. Rolleston. LC. CT: Frenz, 35-37.

July 18. From James R. Osgood \& Company. LC. PT: $C W, 8: 283$.

August 30. From John Boyle O'Reilly (enclosing WW's article in the Boston Pilot). LC.

September 13. From James R. Osgood \& Company. LC. PT: $C W, 8: 284$.

September 17. From T. W. H. Rolleston, LC. CT: Frenz, 38-42.

September 21. From John Boyle O'Reilly. Location unknown. ст: Traubel, 2:136.

September 28. From John Ward Dean. Fragment in Daybooks, 262.

October 5. From Standish James O'Grady. ст: Traubel, 1:399-400.

November 4. From Louis Karpstyin(?) (autograph request). LC.

November 14. From Benjamin Ticknor. LC. PT: $C W, 8: 287$.

November 27. From Rudolf Schmidt.

November 28. From John Fitzgerald Lee. LC. CT: Frenz, 48-50.

November 28. From T. W. H. Rolleston. LC. CT: Frenz, 43-47.

November. From Hannah Heyde. LC. ст: Life in Letters: American Autograph Fournal, 2 (1939): 104-6.

December 6. From Thomas Nicholson.

December 10. From Benjamin Ticknor. LC. PT: $C W, 8: 286-87$.

December 13. From James R. Osgood \& Company. LC.

December 14. From Anne Gilchrist. LC. Ст: Harned, 203-4.

December 31. From R. M. Bucke. ст: Lozynsky, 10.

\section{2}

January 7. From T. W. H. Rolleston. LC. Ст: Frenz, 54-55.

January 11. From Joseph M. Stoddart. LC. CT: Barrus, 235n.

January 15. From Herbert Gilchrist.

January 25. From John H. Johnston. LC. PT: $C W, 8: 288$.

January 29-February 6. From Anne Gilchrist. LC. Ст: Harned, 205-6.

February 14. From T. W. H. Rolleston. LC. ст: Frenz, 56-60. 
March 1. From Oscar Wilde. ст: Traubel, 2:288; The Letters of Oscar Wilde, ed. Rupert Hart-Davis (1962), 99-100.

March 4. From James R. Osgood \& Company. LC. ст: $C W, 8: 289$.

March 16. From Edward Carpenter. ст: Traubel, 1:252.

March 20. From James R. Osgood \& Company. LC. ст: $C W, 8: 292$.

March 21. From James R. Osgood \& Company. LC. PT: $C W, 8: 293-94$.

March 29. From James R. Osgood \& Company. LC. PT: $C W, 8: 295$.

April 10. From James R. Osgood \& Company. LC. ст: $C W, 8: 296$.

April 13. From James R. Osgood \& Company. LC. ст: $C W$, 8:297.

April 23. From H. S. Kneedler, an admirer. LC.

May 1. From John Burroughs. ст: Traubel, 3:350-51; Barrus, 211-212.

May 4. From James R. Osgood \& Company. LC. ст: $C W, 8: 298$.

May 8. From Anne Gilchrist. LC. CT: Harned, 207-8.

May 9. From R. M. Bucke. LC (Bucke's transcription). ст: Lozynsky, 11.

May 9. From William D. O'Connor. Syracuse. ст: Traubel, 4:433-34.

May 9. From Joseph M. Stoddart. LC.

May 11. From Jacob Moller (autograph request). LC.

May 20. From William D. O'Connor. ст: Traubel, 2:12-15.

May 20. From Benjamin Ticknor, for Osgood \& Company. LC.

May 21. From Mrs. Jenny C. Croly (requesting a poem for Demorest's Illustrated). LC.

May 21. From Helen Wilmans. ст: Traubel, 1:49-50.

May 22-25. From Alfred Janson Bloor.

May 23. From John C. Everrett, a student.

May 25. From Benjamin Ticknor, for Osgood and Company. LC. PT: $C W$, 8:299.

May 25. From Benjamin R. Tucker. ст: Traubel, 2:253-54.

May 26. From Fred R. Guernsey, of The Boston Herald.

May 28. From Van Doran Stafford.

May 29. From William D. O'Connor. ст: Traubel, 3:282-84.
May 29. From John G. Willson (requesting WW's presence at the "Melancholy Club").

June (?). From W. S. Kennedy.

June 3. From George W. Christy, an offended reader. LC.

June 3. From William D. O'Connor. ст: Traubel, 1:52-54.

June 4. From Edwin H. Woodruff (enclosing a poem).

June 5. From Rees Welsh \& Company. Pennsylvania.

June 10. From T. W. H. Rolleston. LC. Ст: Frenz, 61-63.

[June 12?]. From R. M. Bucke. ct: Lozynsky, 12.

June 15. From William D. O'Connor.

June 16. From John Burroughs. ст: Traubel, 2:171-72; Barrus, 217.

June 16. From Rees Welsh \& Company. Pennsylvania.

June 18. From Anne Gilchrist. Pennsylvania.

June 19. From William D. O'Connor. ст: Traubel, 1:312-14.

June 21. From Rees Welsh \& Company. Pennsylvania.

June 24. From William D. O'Connor. ст: Traubel, 3:349.

June 26. From Rees Welsh \& Company. Pennsylvania.

June 29. From William D. O'Connor. ст: Traubel, 3:48-50.

July 5. From Rees Welsh \& Company. Pennsylvania.

July 7. From William D. O'Connor.

July 10. From William D. O'Connor.

July 13. From William D. O'Connor.

July 20. From William D. O'Connor. ст: Traubel, 2:60-61.

July 25. From Richard Worthington. Yale.

July 27. From George Chainey.

August 2. From Ainsworth R. Spofford. LC.

August 9. From John Burroughs.

August 12. From John Swinton. ст: Traubel, 2:393.

August 14. From T. W. H. Rolleston. LC. Ст: Frenz, 64-66.

August 16. From Herbert Gilchrist. ст: Traubel, 1:227-29. 
August 19. From William D. O'Connor. ст: Traubel, 2:495-97.

August 24. From John Burroughs.

August 28. From William D. O'Connor.

September 20. From William D. O'Connor. CT: Traubel, 4:20-21.

September 24. From T. W. H. Rolleston. LC. CT: Frenz, 67-68.

October 11. From R. M. Bucke. cr: Lozynsky, 13-14.

October 18. From James Arnold (ordering a book).

October(?) 20. From Herbert Gilchrist.

October 23. From W. Hale White (ordering a book).

October 26. From William D. O'Connor.

October 27. From William D. O'Connor. Yale. ст: Traubel, 4:322-23.

October 29. From John Burroughs. ст: Traubel, 1:333-34; Barrus, 232-33.

October 29. From Thomas Jefferson Whitman. ст: Berthold and Price, 17578.

October 29. From T. W. H. Rolleston. LC. CT: Frenz, 69-70.

November 3. From Rudolf Schmidt.

November 5. From Ezra H. Heywood. Berg.

November 9. From R. M. Bucke. ст: Lozynsky, 15.

November 21. From Edward Dowden. cT: Traubel, 2:363.

November 24. From Anne Gilchrist. LC. cт: Harned, 209-10.

December 10. From R. M. Bucke. cT: Lozynsky, 16.

December 16. From Charles de Kay (requesting that WW join a literary society).

December 18. From William D. O'Connor.

December 19. From Mrs. Elisa S. Leggett. Location Unknown. ст: Donaldson, 246-48.

December 19. From William D. O'Connor.

December 26. From T. W. H. Rolleston. LC. ст: Frenz, 73-76.

\section{3}

January 9. From G. C. Macaulay. ст: Traubel, 2:62.
January 27-February 13. From Anne Gilchrist. LC. ст: Harned, 211-12.

February 3. From John Russell Young. Ст: Traubel, 3:311.

February 20. From William D. O'Connor. ст: Traubel, 1:350-53.

February 23. From Robert Pearsall Smith.

February 25. From John Burroughs. Location unknown. Cited in A List of Manuscripts, Books ... from the Whitman Collection of Mrs. Frank fulian Sprague (1939), 40.

March 10. From William D. O'Connor.

March 12. From R. M. Bucke. cr: Lozynsky, 17.

March 14. From William D. O'Connor. Syracuse. ст: Traubel, 4:406-7.

March 14. From D. L. Proudfit (ordering books).

March 15. From William D. O'Connor. Syracuse. ст: Traubel, 4:459-60.

March 17. From William D. O'Connor. Ст: Traubel, 4:354-56.

March 18. From R. M. Bucke. Feinberg:LC. Ст: Lozynsky, 17-18.

March 19. From William D. O'Connor.

March 20 (letter). From R. M. Bucke. ст: Lozynsky, 19.

March 20 (post card). From R. M. Bucke. ст: Lozynsky, 18-19.

March 25. From Albert G. Knapp, an ex-soldier (ordering a book). ст: Shively (2), 154-55.

March 27. From R. M. Bucke. cт: Lozynsky, 19-20.

March 27. From William D. O'Connor. ст: Traubel, 3:563-66.

April 1. From William D. O'Connor. ст: Traubel, 2:258-61.

April 4. From William D. O'Connor. ст: Traubel, 1:67-68.

April 8. From William D. O'Connor.

April 9. From William D. O'Connor. ст: Traubel, 4:138-39.

April 17. From William D. O'Connor. Syracuse. ст: Traubel, 4:90-92.

April 23. From V. D. Davis, a youthful admirer.

April 29. From Herbert Gilchrist. Lc. ст: Harned, 213-14.

April 30. From Craig Maginnis, an admirer. 
May 6. From Anne Gilchrist. LC. Ст: Harned, 215-16.

May 9. From R. M. Bucke. ст: Lozynsky, 21.

May 20. From John Burroughs. Syracuse. ст: Traubel, 4:509-10.

May 23. From William D. O'Connor. Lion.

May 28. From R. M. Bucke. ст: Lozynsky, 21.

June 2. From R. M. Bucke. ст: Lozynsky, 22.

June 7. From Richard Watson Gilder. ст: Traubel, 2:165-66.

June 8. From T. H. Bartlett. LC (verso of trial lines for "Drift Sands").

June 13. From Elizabeth Ford (ordering books).

June 13. From John H. Johnston.

June 15. From William D. O'Connor. Syracuse. Ст: Traubel, 4:162 (dated June 13).

June 16. From Hannah Heyde. Lc.

June 20. From Arthur Boyle, for the Santa Fé Tertio Millennial Celebration (requesting a poem).

June 26. From William Roscoe Thayer (asking about a letter written by Sidney Lanier).

July 12. From William D. O'Connor. ст: Traubel, 3:128-30.

July 14. From Hannah Heyde. Lc.

July 20. From William D. O'Connor. ст: Traubel, 3:130-31.

July 20. From John Swinton. ст: $A L, 39$ (1968):550.

August 17. From John Burroughs. ст: Traubel, 1:403.

August 17. From William D. O'Connor.

August 22. From Charles Scribner's Sons. Princeton: Scribner Archives.

August 27-October 22. From Herbert Gilchrist.

September 9. From R. M. Bucke. ст: Lozynsky, 23.

September 14. From Karl Knortz. Yale.

September 18. From William D. O'Connor. Syracuse. Ст: Traubel, 4:394-95.

September 21. From John Burroughs.

September 22. From Charles W. Eldridge. Yale.

September 22. From William D. O'Connor. Ст: Traubel, 4:191-92.
September 23. From R. M. Bucke. ст: Lozynsky, 24.

September 24. From William D. O'Connor.

September 27. From T. W. H. Rolleston. LC. CT: Frenz, 77-79.

September 30. From T. W. H. Rolleston. October 13. From T. W. H. Rolleston.

October 13-21. From Anne Gilchrist. LC. ст: Harned, 220-22.

October 18. From Charles L. Heyde. Trent.

November 17. From T. F. Macdonald. Syracuse. ст: Traubel, 4:197-98.

November 18. From John Burroughs. Syracuse. ст: Traubel, 4:35-37.

November 20. From H. N. Whitman (acknowledging receipt of a book and requesting genealogical information).

November 22. From T. W. H. Rolleston. Syracuse. ст: Traubel, 4:111-12.

November 23. From Emma Riley (requesting Specimen Days).

November 28. From Harry Stafford. ст: Shively (1), 170.

November 30. From Henry M. Alden, for Harper's New Monthly Magazine. ст: Traubel, 2:220.

December 17. From Harry Stafford. ст: Shively (1), 170-71.

December 23. From Mary Van Nostrand. Yale.

\section{4}

January 1. From T. W. H. Rolleston. LC. Ст: Frenz, 80-82.

January 4. From Alice G. Brown (autograph request). LC.

January 8. From John Burroughs. ст: Traubel, 1:395; Barrus, 247.

January 8. From General William J. Sewell. Mrs. Doris Neale.

February 6. From Josephine Barkeloo (thanking WW for an article). LC.

February 8. From Thomas G. Gentry. University of Iowa. ст: $W W Q R, 1$, no. 1 (1983):18. See letter 1262.5.

February 10. From Harry Stafford. ст: Shively (1), 171.

February 16. From W. S. Kennedy. Yale.

February 18. From W. S. Kennedy. LC. 
February 22. From William D. O'Connor. Yale.

February 27. From Frederick W. Wilson, LC.

March 2. From Edward Carpenter. Lion. ст: Traubel, 4:76-77.

March 4. From T. W. H. Rolleston. LC. Ст: Frenz, 83-86.

March 12. From Allen Upward. Syracuse. Ст: Traubel, 4:212-17.

March 25. From John H. Johnston. СT: Traubel, 3:331.

April 5. From Anne Gilchrist. LC. ст: Harned, 223-24.

April 5. From T. W. H. Rolleston. LC. CT: Frenz, 87-88.

May 2. From Anne Gilchrist. LC. CT: Harned, 225-26.

May 5. From T. W. H. Rolleston. LC. CT: Frenz, 90-91.

May 11. From Eleanor M. Lawney, an admirer.

May 26. From S. W. Foss, of the Lynn (Mass.) Saturday Union. ст: Traubel, 2:227.

May 31. From Harry Tyrrel.

June 3. From Mrs. Mattie Maxim (ordering a book). LC.

June 8. From T. H. Bartlett. LC.

June 10. From Dr. J. W. Bartlett (payment for a book). LC.

June 10. From Folger McKinsey (autograph request).

June 23. From George W. Ludwig (inquiring about price of WW's books). LC.

June 25-26. From Mrs. M. B. Minchen, mystical admirer.

June 27. From L. Birge Harrison (praising Specimen Days). LC.

June 30(?). From L. Birge Harrison.

July 12. From Robert Underwood Johnson. ст: Traubel, 2:218.

July 16. From Harry W. Gustafson, young admirer.

July 21. From Anna M. Wilkinson (acknowledging receipt of books).

July 30. From Parker Pillsbury (acknowledging receipt of book). LC.

August 5. From Anne Gilchrist. LC. ст: Harned, 227.

August 7. From T. W. H. Rolleston. Syracuse. ст: Traubel, 4:113-14.
August 9. From Richard Watson Gilder. CT: Traubel, 2:212.

August 11. From E. V. Garrison (invitation to spend the evening). LC.

August 16. From Pliny B. Smith (ordering books). LC.

August 20. From James Knowles, editor of The Nineteenth Century (requesting a manuscript).

August 24. From Edwin Booth. cr: Traubel, 1:355; WWR, 6 (1960):50.

August 28. From Edwin Booth. cT: Traubel, 1:46; WWR, 6 (1960):50.

September 5. From Robert Pearsall Smith. LC.

September 9. From T. W. H. Rolleston. Ст: Traubel, 1:18-21; Frenz, 94-97.

September 17. From Cupples, Upham \& Company (ordering a book).

September 29. From R. Watson (requesting an inscribed book). LC.

September 30. From Herbert Gilchrist.

September 30. From The Critic, enclosing a check. LC. See NUPM, 6:2105.

October 2. From William D. O'Connor. Ст: Traubel, 1:177-79.

October 14. From Charles L. Heyde. Trent.

October 16(?). From William C. Bryant. LC.

October 20(?). From Hannah Heyde. Lc.

October 22. From Henry M. Alden. ст: Traubel, 2:218-19.

October 26. From Anne Gilchrist. LC. сT: Harned, 228-29.

November 1. From Frederick York Powell. ст: Traubel, 1:356-57.

November 2. From Charles L. Heyde. Trent.

November 12. From Mary Whitall Smith. PT: Smith Alumnae Quarterly (February 1958): 86-87.

November 14. From James Matlack Scovel. LC.

November 26. From Charles L. Heyde. Trent.

November 28. From John Addington Symonds. ст: Schueller and Peters, 2:97274.

December 17. From Anne Gilchrist. LC. cT: Harned, 230-32.

December 20. From Charles L. Heyde. Trent. 
December 21. From Mary B. N. Williams (inviting WW to dinner).

December 29. From Edmund Gosse. ст: Traubel, L:40 (dated 1887); Victorian Studies, 1 (1957): 181.

December 29. From John(?) B. Robinson, for the Delaware County Institute of Science (requesting a lecture).

[1884(?)]. From Charles W. Dailey (autograph request).

\section{5}

January 1. From William Michael Rossetti. Ст: Traubel, 1:436-37; Peattie, 462-63.

January 7. From W. S. Kennedy.

January 9. From A. B. Drake (acknowledging receipt of books).

January 13. From Henry M. Alden. Ст: Traubel, 2:211 (dated January 3).

January 16. From W. S. Kennedy.

January 17. From Charles L. Heyde. Trent.

January 24. From Charles L. Heyde. Trent.

February 1. From William D. O'Connor. CT: Traubel, 1:29-33.

February 5(?). From William C. Skinner (payment for a book). LC.

February 11. From John Boyle O'Reilly. Cт: Traubel, 1:8-9

February 11. From T. W. H. Rolleston. Cт: Traubel, 3:85-86; Frenz, 99-100.

February 13. From John Swinton. ст: Traubel, 3:271.

February 19. From Charles L. Heyde. Trent.

February 20. From Professor George Herbert Palmer. ст: Traubel, 1:112.

February 23. From Thomas Jefferson Whitman. ст: Berthold and Price, 17982.

February 27. From Anne Gilchrist. LC. CT: Harned, 233-35.

February 27. From Charles L. Heyde. Trent. ст: Gohdes and Silver, 228-29.

March 5. From John Boyle O'Reilly. ст: Traubel, 2:38.

March 7. From William D. O'Connor. Syracuse. ст: Traubel, 4:477-78.

March 10. From Gabriel Harrison (acknowledging receipt of a book).
[March 11]. From W. S. Kennedy.

March 16. From Charles L. Heyde. Trent.

March 17. From Palin H. Sims, an exsoldier (recalling Civil War experiences).

March 18. From Ellen M. Abdy-Williams (concerning receipt of books).

March 31. From George Parsons Lathrop (inviting WW to give readings to raise funds in aid of international copyright).

April 5. From R. M. Bucke. ст: Lozynsky, 25-26.

April 7. From James Matlack Scovel.

May 4. From Anne Gilchrist. LC. Ст: Harned, 236-38.

May 7. From James Matlack Scovel.

May 11. From M. Schuyler(?), of Harper's Weekly.

May 12 . From Henry M. Alden. cr: Traubel, 1:61.

May 12. From James Matlack Scovel.

May 18. From John Burroughs.

May 21. From Samuel B. Wright (inquiring about WW's books).

May 31. From Ernest Rhys.

[June 2]. From W. S. Kennedy.

June 8. From Henry M. Alden. ст: Traubel, 1:28.

June 9. From Robt. Lutz (inquiring about a German translation of Leaves of Grass).

June 10. From Carolan O'Brien Bryant (ordering books).

June 13. From George Weaver (autograph request).

[About June 16] (Monday). From James Matlack Scovel.

[About June 16] (Thursday). From James Matlack Scovel.

June 21-22. From Anne Gilchrist. LC. CT: Harned, 239-40 (dated January 21).

June 23. From Charles L. Heyde. Trent.

June 30. From James Redpath. ст: Traubel, 2:73-74.

July 1. From William J. Linton. ст: Traubel, 2:442-43.

July 7. From Ernest Rhys. CT: Traubel, 1:451-53.

July 8. From Charles Aldrich (autograph request). LC.

[July] 9. From Edward Carpenter (misdated June 9). 
July 14. From Charles Allen Thorndike Rice. Syracuse. ст: Traubel, 4:329-30.

July 16. From James Redpath. ст: Traubel, 2:74-75.

July 20. From Anne Gilchrist. LC. ст: Harned, 241-42.

July 21. From Herbert Gilchrist.

July 25. From William D. O'Connor. Syracuse. ст: Traubel, 4:67-70.

July 25. From Charles Parsons, of Harper's Weekly (requesting permission to publish WW's protrait).

July 25. From Mary Whitall Smith. Boston University. ст: Donaldson, 234-36.

July 30. From Charles L. Heyde. Trent.

July 31. From Alexander K. Reamer, a rhapsodic admirer.

July 31. From Thomas Jefferson Whitman. ст: Berthold and Price, 182-83.

August 4. From T. W. H. Rolleston. ст: Traubel, 3:487-88; Frenz, 101-3.

August 8(?). From W. S. Kennedy.

August 11. From James Redpath. CT: Traubel, 2:75-76.

August 15. From James Matlack Scovel.

August 17. From Charles W. Eldridge.

August 17. From Edw. Mawson (relating theatrical reminiscences).

[August 28]. From W. S. Kennedy.

August 28. From William Michael Rossetti. Hanley. ст: Peattie, 474.

August [30]. From John Newton Johnson.

September 5. From Herbert Gilchrist.

September 5. From an unidentified correspondent (asking for a synopsis of a forthcoming book).

September 9. From John H. Johnston.

September 11. From Thomas Jefferson Whitman. Yale. ст: Berthold and Price, 183.

September 15. From R. M. Bucke. cT: Lozynsky, 27.

September 16. From A. B. Nourse (asking the price of a book).

September 19. From Houghton, Mifflin \& Company (inquiring about the first edition of Leaves of Grass).

September 25-29. From Ernest Rhys. PT: Traubel, 3:162-64.

September 29. From Herbert Gilchrist.

October 2. From R. M. Bucke.
October 4. From William Michael Rossetti. Syracuse. CT: Traubel, 4:209; Peattie, 475-76.

October 6. From William Michael Rossetti. ст: Traubel, 3:65-66.

October 7. From John Burroughs. ст: Traubel, 1:404; Barrus, 257.

October 12. From William Roscoe Thayer.

October 15. From Richard A. Stuart (inquiring whether WW was willing to read his poetry at a meeting).

October 20. From Lionel Johnson. Ст: Traubel, 2:180-81.

October 20. From James Redpath. ст: Traubel, 2:232.

October 23. From Edward Carpenter. ст: Traubel, 3:192-93.

October 23. From James Redpath.

November 13. From William Michael Rossetti. ст: Traubel, 2:330-31; Peattie, 478-79.

November 17. From Lorenz Reich, an admirer.

November 18. From Herbert Gilchrist.

[November(?)]. From Karl Knortz. ст: Traubel, 3:488-89 (undated).

December 2. From Herbert Gilchrist.

December 2. From W. S. Kennedy. ст: Traubel, 4:479-80.

December 6. From Marion Thrasher (requesting that WW tour the midwest giving readings at $\$ 50$ a night).

December 31. From John Burroughs. PT: Traubel, 2:86-87; Barrus, 258-59.

December. From B. P. Steward, a young admirer.

\section{6}

[January 2?]. From W. S. Kennedy. Berg. January 5. From William Michael Rossetti. CT: Traubel, 2:291; Peattie, 48182.

January 11. From William Michael Rossetti.

January 21. From William D. O'Connor. ст: Traubel, 3:74-75.

January 25. From Herbert Gilchrist.

February 2. From John Newton Johnson.

February 5. From W. S. Kennedy.

March 23. From William D. O'Connor. Syracuse. ст: Traubel, 4:413-15. 
March 26. From Hiram Corson. cT: Traubel, 1:286-87.

March 30. From Roden Noel. CT: Traubel, 1:432-33.

April 3. From John Burroughs. ст: Traubel, 2:549-50.

April 9. From Moses A. Walsh (concerning the Wesley Water Cure).

April 12. From Smith \& Smith, managers of the Salem (N.J.) Opera House (proposing a Lincoln lecture).

April 15. From Talcott Williams.

April 19. From W. S. Kennedy.

April 26. From Hiram Corson. ст: Traubel, 1:287.

May 13. From R. M. Bucke. ст: Lozynsky, 28.

May 16. From Roden Noel. ст: Traubel, $1: 394$.

May 17. From Edward Carpenter.

May 17. From Dr. John Johnston.

May 17. From William Michael Rossetti.

May 22. From Ernest Rhys. Hanley.

May 25. From William D. O'Connor. Syracuse. ст: Traubel, 4:283-85.

June 9. From R. M. Bucke. Barrett. ст: Lozynsky, 29.

June 11. From Talcott Williams.

June 14. From W. I. Whiting, for Scammel Brothers (noting auction prices for WW items).

June 14. From Lavinia F. Whitman (concerning Whitman genealogy).

June 17. From W. S. Kennedy.

June 19. From Edward T. Potter. Syracuse. ст: Traubel, 4:311-12.

June 29. From John Burroughs.

July 1. From W. S. Kennedy.

July 5. From Gertrude Van Duren (ordering a book).

July 7. From W. L. Shoemaker, an admirer.

July 10 . From W. S. Kennedy.

July .13. From Samuel G. Stanley, a Brooklyn friend.

July 16. From Elizabeth J. Sharpe, a friend of the Staffords.

July 17. From George M. Williamson (offering to purchase WW manuscripts).

July 18. From W. S. Kennedy. LC.
July 19. From Charles Morris (requesting permission to include "Song of the Redwood-Tree" in Half Hours with the Best American Authors).

July 30. From Mary Grace Thomas, a student at Bryn Mawr College.

August 2. From W. S. Kennedy.

August 17. From William D. O'Connor. Yale.

August 18. From W. S. Kennedy.

September 10. From Herbert Gilchrist.

September 16. From James Matlack Scovel.

September 16. From William S. Walsh, of Lippincott's.

September 20. From Henry M. Alden, of Harper's Monthly. ст: Traubel, 2:226.

October 5. From James Redpath, of The North American Review. ст: Traubel, 2:226.

October 5. From Charles F. Wingate, secretary of The Twilight Club in New York.

October 16. From Herbert Gilchrist.

October 18. From W. I. Whiting (concerning a recent auction).

October 21. From Mary Smith Costelloe. PT: Smith Alumnae Quarterly (February 1958):87.

October 21. From Percy Ives, the artist. ст: Detroit Historical Society Bulletin, 16 (February 1960): 9.

November 9. From Herbert Gilchrist.

November 9. From Thomas Jefferson Whitman. Ст: Berthold and Price, 184.

November 13. From James Wilkie (enclosing a gift).

November 16. From William Michael Rossetti.

November 24. From Hamlin Garland. Ст: Traubel, 2: 160-62; Garland, Roadside Meetings (1930), 128-30; WWQR, 5, no. 3 (Winter 1988):2-5.

November 26. From Ernest Rhys. CT: Traubel, 4:228-30.

December 4. From R. M. Bucke. ст: Lozynsky, 30-31.

December 6. From Sylvester Baxter (concerning a pension).

December 10. From William D. O'Connor. Syracuse. CT: Traubel, 4:128-30.

December 17. From Gerald Maxwell, a young admirer. 
December 21. From John Burroughs. Yale. ст: Traubel, 4:130-31.

December 21. From William D. O'Connor.

December 23. From Herbert Gilchrist.

December 25. From Morley C. Roberts. ст: Traubel, 3:466-67 (dated December 12).

\section{7}

January 6. From Herbert Gilchrist.

January 11. From the Editor of the New Orleans Picayune. Location unknown. Cт: PW, 605.

January 15. From Alfred Tennyson. cT: Corr., 4:63-64.

January 15. From Percy W. Thompson (autograph request).

January 17. From Mary Smith Costelloe. PT: Smith Alumnae Quarterly (February 1958):87.

January 19. From Ernest Rhys (WW's notation: "a wonderful letter").

February 1. From R. Brisbane. Syracuse. CT: Traubel, 4:266-67.

February 3. From Henry Norman, of Pall Mall Gazette.

February 3. From Louis H. Sullivan. ст: Traubel, 3:25-26; Sherman Paul, Louis Sullivan: An Architect in American Thought (1962), 1-3.

February 11. From Charles W. Eldridge. Location unknown. ст: Barrus, 262-63.

February 12. From C. A. Spofford (requesting information about Leaves of Grass). LC.

February 15. From Ernest Rhys. Morgan.

February 16. From Joseph B. Marvin. Syracuse. ст: Traubel, 4:412.

February 20. From R. M. Bucke. ст: Lozynsky, 32.

February 28. From Daniel G. Brinton.

March 11. From Mrs. William Hawley Smith.

March 12. From John Hay. Location unknown. ст: Traubel, 3:91-92.

March 13. From Annie Fields (extending an invitation on behalf of an unnamed committee).

March 16. From Edward W. Bok (concerning a memorial for Henry Ward Beecher).
March 17. From Joseph B. Gilder. Barrett. ст: The Princeton University Library Chronicle, 3 (November 1941):6.

March 29. From John H. Johnston. ст: Traubel, 2:431.

March 29. From Ernest Rhys. Barrett: Virginia.

March 31. From Herbert Gilchrist.

April 8. From James Grant Wilson. ст: Traubel, 2:135.

April 9. From W. S. Kennedy.

April 19. From William Carey, of The Century Magazine (requesting an autographed copy of "O Captain! My Captain!"). LC.

April(?) 19. From Charles L. Heyde. Trent.

April 20. From Edward Carpenter.

April 25. From Major James B. Pond.

April 29. From Ernest Rhys. Location unknown. CT: Traubel, 4:487-88.

April 30. From Frederick A. Stokes, a publisher.

May 15(?). From Dr. John Johnston and James W. Wallace. Typescript in Bolton.

May 19. From James Knowles. ст: Traubel, 1:28.

May 24. From Ernest Rhys. ст: Traubel, 3:59-61.

May 27. From Herbert Gilchrist.

June 1. From George M. Williamson. Barrett.

June 2. From John W. Worth (concerning his trip West).

June 12. From R. M. Bucke. ст: Lozynsky, 33.

June 13. From Fred G. Kitton (asking for WW's impressions of Charles Dickens).

June 16. From Alfred Emery. LC.

June 16. From W. S. Kennedy. Boston Public Library.

June 18. From Sylvester Baxter. LC.

June 21. From Sylvester Baxter. cT: Traubel, 2:305.

July 7. From Grace Ellery Channing.

July 9. From Cassius M. Clay (acknowledging receipt of a book and including an address he delivered at Yale).

July 29. From William F. Channing. LC.

July 31. From Nugent Robinson, of Once $A$ Week. ст: Traubel, 2:220 (dated July 20). 
August 1. From William Morlow Fullerton (acknowledging WW's gifts).

August 2. From Sylvester Baxter. Syracuse. ст: Traubel, 2:378-79.

August 2. From Ellen M. O’Connor.

August 2. From Louisa Snowdon. Location unknown. ст: Traubel, 4:233-34.

August 3. From Hampton L. Carson, of the Constitutional Centennial Commission (requesting a patriotic poem for the occasion).

August 5. From Percy Ives. ст: Detroit Historical Society Bulletin, 16 (February 1960):10.

August 9. From the City Surveyor of Camden.

August 10. From James Redpath (requesting an article for The North American Review). Historical Society of Pennsylvania.

September 2. From Walter Lewin (enclosing his article on WW in Murray's Magazine).

September 10. From John H. Johnston.

[September 12]. From Sidney H. Morse.

September 24. From James Matlack Scovel (asking WW to write on the Irish question). LC.

October 8. From Sylvester Baxter. Location unknown. СT: Traubel, 2:299-300.

October 10. From Herbert Gilchrist.

November 3. From A. H. Spaulding (reporting his defense of WW's "Children of Adam" before a Boston literary society).

November 15. From Alfred Tennyson. Yale. ст: Corr., 4:131.

November 16. From Herbert Gilchrist.

November 18-22. From W. S. Kennedy.

November 30. From M. H. Spielmann. CT: Traubel, 2:232-33.

December 5. From W. S. Kennedy.

December 11. From Julius Chambers. Location unknown.

December 11. From Thomas Jefferson Whitman. ст: Berthold and Price, 18586.

December 26. From Sidney H. Morse.

December 30. From Mrs. Anna M. Kerr (recalling old Brooklyn days).
January 2. From W. S. Kennedy.

January 3. From William D. O'Connor.

January 4. From Ernest Rhys.

January 4. From Ellen Terry. ст: Traubel, $1: 5$.

January 10. From W. S. Kennedy (misdated 1887).

January 11. From W. S. Kennedy (misdated 1887).

January 13. From John Burroughs.

January 13. From John Greenleaf Whittier. ст: Traubel, 2:8.

January 23. From James Gordon Bennett. LC.

January 26. From Ernest Rhys. Syracuse. ст: Traubel, 4:47-48.

January 30. From Ellen M. O'Connor.

January 31. From Charles H. Buck (autograph request).

January 31. From Sidney H. Morse.

[February 8?]. From W. S. Kennedy.

[February 10?]. From R. M. Bucke. Berg. ст: Lozynsky, 34 .

February 12. From Richard W. Colles. Syracuse. ст: Traubel, 4:141-42.

February 13-14. From W. S. Kennedy. Berg.

February 16. From Mrs. Talcott Williams

February 17. From Herbert Gilchrist.

February 20. From Ernest Rhys. PT: Barrus, 273n.

February 21. From R. M. Bucke. ст: Lozynsky, 34-35.

February 22. From Sidney H. Morse.

[February 25]. From W. S. Kennedy.

February 26. From Sidney H. Morse.

March 1(?). From W. S. Kennedy. Yale.

March 2. From Judah B. Voorhees, of the Society of Old Brooklynites.

March 3. From Charles T. Sempers, a Harvard student.

March 4. From Charles T. Sempers.

March 6. From W. J. Hemsley (enclosing a sonnet).

March 7. From Ernest Rhys. Lion. ст: Traubel, 4:46-47.

March 8. From John R. Witcraft, a young admirer. Barrett.

March 11. From R. M. Bucke. ст: Lozynsky, 36.

March 13. From Ellen M. O'Connor.

March 14. From Sidney H. Morse. 
March 20. From Henry H. Collins, an admirer.

March 24. From John W. Wiggins, of the Society of Old Brooklynites. Harvard.

March 29. From W. S. Kennedy.

April 3. From Ernest Rhys. ст: Traubel, 2:30-31.

April 7. From James Gordon Bennett.

April 7. From Thomas J. McKee.

April 13. From Sheridan Ford. Syracuse. CT: Traubel, 4:496-97.

April 14. From William D. O'Connor. Syracuse. ст: Traubel, 4:497-99.

April 19. From Hamlin Garland. ст: $W W Q R$, 5, no. 3 (Winter 1988):5-6.

[April 22]. From W. S. Kennedy.

April 25. From R. M. Bucke. Yale. ст: Lozynsky, 37.

April 26. From Francis Vielé-Griffin. Location unknown. ст: Traubel, 1:119.

April 28. From O. G. Hempstead \& Son. Location unknown. ст: Traubel, 1:93.

May 4. From James Gordon Bennett. Yale.

May 13. From William Harrison Riley.

May [15?]. From Dr. John Johnston and James W. Wallace. Typescript in Bolton.

May 16. From William D. O'Connor. Syracuse. ст: Traubel, 4:244-47.

May 21. From Ernest Rhys. ст: Traubel, 1:292-93.

May 30. From Ernest Rhys. cт: Traubel, 2:31-32.

May 31. From F. S. Ryman (birthday greetings).

May. From Ingersoll Lockwood (sending The American Bookmaker for August 6 with an article about WW and a portrait by Frank Fowler).

June 7. From Ernest Rhys. ст: Traubel, $2: 33$

June 7. From George M. Williamson.

June 8. From W. S. Kennedy.

June 11. From Edmund Blake (autograph request).

June 11. From John Burroughs. CT: Traubel, 1:418-19; Barrus, 274-75.

June 13. From William D. O'Connor. Syracuse. ст: Traubel, 4:499-500.

June 13. From Lt. Percy Thompson (autograph request).
June 15. From R. M. Bucke. ст: Lozynsky, 38-39.

June 15. From Sidney H. Morse. ст: Traubel, 1:405-6.

June 16. From Elmer B. Lane (autograph request).

June 18. From William Hosen Ballou (concerning WW's pension).

June 20. From Robert Pearsall Smith.

June 23. From Charles F. Sloane, a young admirer.

July 2. From Reginald A. and Katie E. Beckett, two fervent English socialists.

July 8. From Herbert Gilchrist.

July 9. From R. M. Bucke. ст: Lozynsky, 40.

July 9. From W. S. Kennedy.

July 9-10. From Ernest Rhys.

July 11. From R. M. Bucke. cт: Lozynsky, 40-41.

July 12. From William D. O'Connor.

July 13. From Sylvester Baxter. ст: Traubel, 2:192-93.

July 14. From Thomas Jefferson Whitman. Ст: Berthold and Price, 187-88.

July 16. From John Burroughs.

July 17. From R. M. Bucke. Gertrude Traubel Collection. ст: Lozynsky, 41.

July 19. From Charlotte Fiske Bates.

July 22. From R. M. Bucke. ст: Lozynsky, 42.

July 24. From R. M. Bucke. ст: Lozynsky, 42-43.

July 24. From C. Sadakichi Hartmann.

July 24. From M. H. Spielmann. CT: Traubel, 2:104.

July 24. From Susan Stafford.

July 25. From William Carey.

July 25. From William D. O'Connor. ст: Traubel, 2:176-77 (dated July 26).

July 27. From R. M. Bucke. ст: Lozynsky, 43-44.

August 3. From William D. O'Connor. CT: Traubel, 2:101-2.

August 4. From John Baker (about WW's illness).

August 4. From R. M. Bucke. ст: Lozynsky, 45.

August 7. From R. M. Bucke. cт: Lozynsky, 45-46.

[August 7]. From Jessie E. Taylor. ст: Traubel, 2:450-51. 
August 8. From R. M. Bucke. cт: Lozynsky, 46-47.

August 10. From R. M. Bucke. ст: Lozynsky, 47.

August 10. From William Ingram. William D. Bailey Collection, Ohio Wesleyan University.

August 11. From R. M. Bucke. ст: Lozynsky, 48.

August 15. From C. H. Browning, of the New York Herald. ст: Traubel, 2:14647.

August 15. From R. M. Bucke. ст: Traubel, 2:147-48; Lozynsky, 48-49.

August 17. From R. M. Bucke. Trent. cT: Lozynsky, 49.

August 21. From Mary Smith Costelloe.

August 21. From John Herbert Clifford (acknowledging receipt of a book).

August 24. From R. M. Bucke. ст: Lozynsky, 50.

August 25. From R. M. Bucke. ст: Lozynsky, 50-51.

August 27. From R. M. Bucke. ст: Lozynsky, 51.

August 28. From R. M. Bucke. ст: Lozynsky, 52.

August 28. From Charles L. Heyde. Trent.

August 29. From Charlotte Fiske Bates. CT: Traubel, 2:263.

August 29. From R. M. Bucke. ст: Lozynsky, 52-53.

August 30. From R. M. Bucke. ст: Lozynsky, 53-54.

August 30. From W. S. Kennedy. ст: Traubel, 2:243.

August 31. From Edward Dowden. ст: Traubel, 3:146-47.

August 31. From William D. O'Connor. Cт: Traubel, 2:238-39.

September 1. From Mary Smith Costelloe.

September 1. From T. W. H. Rolleston. Barrett.

September 2. From R. M. Bucke. ст: Lozynsky, 55.

September 2. From Sidney H. Morse. ст: Traubel, 3:387-88.

September 3. From R. M. Bucke. ст: Lozynsky, 55-56.

September 3. From Mrs. C. F. Stowe, a loving admirer.
September 3. From Mrs. Mary B. N. Williams.

September 4. From R. M. Bucke. CT: Lozynsky, 56.

September 4(?). From W. S. Kennedy. Ст: Traubel, 2:263-64.

September 7. From Logan Pearsall Smith.

September 7. From R. M. Bucke. cr: Lozynsky, 56-57.

September 8. From R. M. Bucke. ст: Lozynsky, 58.

September 9. From R. M. Bucke. ст: Lozynsky, 58-59.

September 10. From R. M. Bucke. ст: Lozynsky, 59.

September 11. From R. M. Bucke. ст: Lozynsky, 60-61.

September 12. From R. M. Bucke. ст: Lozynsky, 61.

September 12. From William Ingram. ст: Traubel, 2:320.

September 14. From R. M. Bucke. ст: Lozynsky, 61.

September 15. From R. M. Bucke. ст: Lozynsky, 61-62.

September 17. From R. M. Bucke. ст: Lozynsky, 62-63.

September 17. From W. S. Kennedy to WW and Horace Traubel.

September 18(?). From James Gordon Bennett.

September 19. From R. M. Bucke. ст: Lozynsky, 63.

September 20. From R. M. Bucke. ст: Lozynsky, 63-64.

September 21. From R. M. Bucke. ст: Lozynsky, 64-65.

[September 21]. From Mrs. C. S. Haley, a gushing admirer.

September 22. From R. M. Bucke. ст: Lozynsky, 65.

September 23. From Julius Chambers.

September 24. From R. M. Bucke. ст: Lozynsky, 66.

September 26. From H. Buxton Forman. CT: Traubel, 2:433-34.

September 26. From Richard J. Hinton.

September 27. From R. M. Bucke. ст: Lozynsky, 66-67.

September 27. From Charles William Dalmon, an English sailor.

September 28. From R. M. Bucke. ст: Lozynsky, 67-68. 
September 30. From R. M. Bucke. ст: Lozynsky, 68.

October 1. From R. M. Bucke. ст: Lozynsky, 69.

October 1. From Mary Smith Costelloe. ст: Smith Alumnae Quarterly (February 1958): 87-88.

October 1. From Herbert P. Horne. ст: Traubel, 2:528.

October 3. From R. M. Bucke. ст: Lozynsky, 69-70.

October 3. From William J. Linton. ст: Traubel, 2:473.

October 5. From R. M. Bucke. ст: Lozynsky, 70.

October 9. From R. M. Bucke. ст: Lozynsky, 71.

[October?] 9. From W. S. Kennedy. ст: Traubel, 2:466.

October 9. From T. W. Mather. ст: Traubel, 2:465.

October 9. From William D. O'Connor. CT: Traubel, 2:467.

October 11. From R. M. Bucke. cT: Lozynsky, 71-72.

October 11. From Ernest Rhys.

October 16. From Jerome Buck. ст: Traubel, 2:501.

October 16. From R. M. Bucke. ст: Lozynsky, 72-73.

October 16. From John Burroughs. Location unknown. ст: Traubel, 2:493-94.

October 18. From Hamlin Garland. ст: Traubel, 2:509; $W W Q R, 5$, no. 3 (Winter 1988):6-7.

[October 18]. From W. S. Kennedy. ст: Traubel, 2:507.

October 19. From Jeannette L. and Joseph B. Gilder.

October 19. From Charles L. Heyde. Trent.

October 20. From W. S. Kennedy.

October 20. From Josiah Child.

October 21. From R. M. Bucke. ст: Lozynsky, 73-74.

October 21. From Logan Pearsall Smith.

October 23. From R. M. Bucke. cт: Lozynsky, 74-75.

October 24. From R. M. Bucke. cT: Lozynsky, 75-76.

October 24. From Hamlin Garland. ст: Traubel, 2:530; WWQR, 5, no. 3 (Winter 1988):7-8.
October 24. From Susan Stafford.

October 25. From R. M. Bucke. cT: Lozynsky, 76.

October 25. From Edmund Clarence Stedman. ст: Traubel, 2:537-38.

October 26. From W. C. Angus. Location unknown. ст: Traubel, 4:198-99.

October 27. From Charles L. Heyde. Trent.

October 27. From William D. O'Connor.

October 28. From R. M. Bucke. ст: Lozynsky, 77.

October 31. From William H. Blauvelt. Location unknown. ст: Traubel, 3:8.

October. From Charles L. Heyde. Trent.

[October?] From Louisa Orr Whitman. Trent.

November 1. From R. M. Bucke. ст: Lozynsky, 78.

November 1. From William D. O'Connor. ст: Traubel, 3:9-10.

November 3. From Wellesley Sayle.

November 4. From R. M. Bucke. ст: Lozynsky, 78-79.

November 6. From R. M. Bucke. ст: Lozynsky, 79-80.

November 8. From R. M. Bucke. ст: Lozynsky, 80-81.

November 9. From R. M. Bucke. ст: Lozynsky, 81.

November 9. From Hamlin Garland. ст: Traubel, 3:67; $W W Q R, 5$, no. 3 (Winter 1988):8-9.

November 11. From R. M: Bucke. ст: Lozynsky, 82-83.

November 14. From R. M. Bucke. ст: Lozynsky, 84.

November 16. From R. M. Bucke. ст: Lozynsky, 84.

November 16. From Hamlin Garland. ст: Traubel, 3:114; $W W Q R, 5$, no. 3 (Winter 1988): 9.

November 17. From Joseph B. Gilder. ст: Traubel, 3:124.

November 22. From R. M. Bucke. ст: Lozynsky, 85.

November 25. From R. M. Bucke. ст: Lozynsky, 86.

November 26. From James Matlack Scovel (concerning a meeting with Maurice Barrymore).

November 28. From R. M. Bucke. ст: Lozynsky, 86-87. 
November 28, Evening. From R. M. Bucke. ст: Lozynsky, 87-88.

November 28. From C. W. Moulton (requesting permission to publish WW's poems in The Magazine of Poetry).

November 29. From George E. Dixon (autograph request). LC.

November 30. From Logan Pearsall Smith.

November. From Charles L. Heyde. Trent.

December 1. From Herbert Gilchrist.

December 2. From R. M. Bucke. ст: Lozynsky, 89.

December 3. From R. M. Bucke. ст: Lozynsky, 89-90.

December 3. From Justin H. McCarthy Jr.

December 6. From R. M. Bucke. ст: Lozynsky, 90-91.

December 7. From R. M. Bucke. ст: Lozynsky, 91.

December [10]. From William D. O'Connor. ст: Traubel, 3:288 (dated December 9).

December 11. From R. M. Bucke. ст: Lozynsky, 92.

December 12. From Ernest Rhys.

December 13. From W. S. Kennedy.

[December 13]. From W. S. Kennedy.

December 15. From R. M. Bucke. ст: Lozynsky, 92-93.

December 15. From Samuel Hollyer.

December 16. From R. M. Bucke. ст: Lozynsky, 93-94.

December 16. From Thomas Tylston Greg. Location unknown. ст: Traubel, 3:432-33.

December 17. From Katherine Johnston.

December 19. From R. M. Bucke. ст: Lozynsky, 94-95.

December 20. From R. M. Bucke. ст: Lozynsky, 95-96.

December 20. From Ellen M. O'Connor.

December 21. From R. M. Bucke. ст: Lozynsky, 96.

December 21. From George M. Williamson.

December 23. From R. M. Bucke. ст: Lozynsky, 97.

December 23. From John Burroughs.

December 24. From R. M. Bucke. cт: Lozynsky, 97-98.
December 25. From Sylvester Baxter.

December 25. From R. M. Bucke. ст: Lozynsky, 99.

December 25. From W. S. Kennedy.

December 25. From Franklin B. Sanborn. Ст: Traubel, 3:402-3.

December 27. From Edward Carpenter.

December 27. From Joseph B. Gilder(?).

December 30. From Elizabeth and Isabella Ford.

December 30. From Sophia Kirk, an admirer.

December 31. From R. M. Bucke. ст: Lozynsky, 100.

\section{9}

January 1. From O. F. Hershey, a Harvard student ("You are no stranger but an ever present comrade").

January 4. From Edwin C. Gellett (sending a pamphlet).

January 5. From Ernest Rhys. ст: Traubel, 3:440-41 (misdated by Rhys and Traubel 1888).

January 5. From Logan Pearsall Smith.

January 6. From Gabriel Sarrazin.

January 7. From Mary Ashley. Location unknown. ст: Traubel, 4:5-6.

January 7. From T. W. H. Rolleston.

January 8. From Frederick York Powell.

January 8. From R. M. Bucke. cT: Lozynsky, 101.

January 9. From R. M. Bucke. cT: Lozynsky, 101-2.

January 9. From O. W. True, an admirer.

[January 11]. From Hamlin Garland. Lion. ст: Traubel, 4:78-79 (dated January 10); $W W Q R, 5$, no. 3 (Winter 1988):9-10.

January 13. From Edward Carpenter.

[January 13]. From Karl Knortz.

January 18. From R. M. Bucke. Trent. ст: Lozynsky, 102.

January 18. From Charles Allen Thorndike Rice. Location unknown. Cт: Traubel, 4:18.

January 20. From R. M. Bucke. Cт: Lozynsky, 103.

January 21 . From W. S. Kennedy.

January 21. From Ellen M. O'Connor.

January 22. From R. M. Bucke. Syracuse. Ст: Lozynsky, 104-5. 
January 25. From R. M. Bucke. ct: Lozynsky, 105.

January 25-26. From Mary Smith Costelloe. PT: Smith Alumnae Quarterly (February 1958):88.

January 26-27. From R. M. Bucke. Syracuse. ст: Lozynsky, 105-6.

January 27. From Edward Carpenter. Syracuse. cT: Traubel, 4:168-69.

January 28. From Ellen M. O'Connor.

January 29. From W. S. Kennedy.

January 29. From John Addington Symonds. Ст: Traubel, 4:125-26; Schueller and Peters, 3:343-44.

January. From Horace C. Simmons (requesting a list of WW's publications for Miss Langley, a bookseller).

February 2. From Ernest Rhys.

February 2. From T. W. H. Rolleston.

February 3. From R. B. Bucke. Lion. ст: Lozynsky, 107.

February 7. From Ellen M. O'Connor.

[February 7]. From R. M. Bucke. Location unknown. PT: Traubel, 4:116; Lozynsky, 107.

February 12. From Joseph B. Gilder.

February 12. From Ellen M. O'Connor.

February 14. From Gabriel Sarrazin. Lion.

[February 15]. From R. M. Bucke. Location unknown. PT: Traubel, 4:163; Lozynsky, 108.

February 17. From R. M. Bucke. Syracuse. ст: Lozynsky, 108-9.

February 21. From John Burroughs. Yale. ст: Traubel, 4:297.

February 22. From W. S. Kennedy.

February 25. From Margaretta L. Avery, WW's cousin. LC.

[February 25]. From W. S. Kennedy.

February 27. From W. S. Kennedy. Yale. ст: Traubel, 4:381.

March 2. From Charles L. Heyde. Trent.

March 2. From Ernest Rhys. Hanley.

March 4. From Gleeson White (requesting information for an English magazine for girls).

March 5. From J. E. Chamberlin of the Boston Evening Transcript. Syracuse. ст: Traubel, 4:320-21.

March 5. From Ellen M. O’Connor. Syracuse. CT: Traubel, 4:292.
March 5. From Huntington Smith (requesting permission to quote from WW's books).

March [6?]. From W. S. Kennedy. Location unknown. PT: Traubel, 4:292.

March 8. From Ellen M. O'Connor. Syracuse. ст: Traubel, 4:309.

March 9. From Miss E. Langley (a book order).

March 12. From Charles L. Heyde. Trent.

March 12. From Ellen M. O'Connor. Syracuse. ст: Traubel, 4:335.

March 13. From Ellen M. O'Connor. Syracuse. ст: Traubel, 4:342.

March 13. From Hannah W. Smith. CT: Smith Alumnae Quarterly (February 1958):88.

March 17. From William S. Walsh, Location unknown. CT: Traubel, 4:372.

March 18. From W. S. Kennedy.

March 18. From Frank H. Williams. Syracuse. ст: Traubel, 4:388.

March 20. From R. M. Bucke. Syracuse. ст: Lozynsky, 110.

March 20. From Ellen M. O'Connor.

March 20. From The Mail and Express (New York) (asking WW's opinion of George Washington).

March 21. From R. M. Bucke. Syracuse. Ст: Lozynsky, 111.

March 21. From Ellen M. O’Connor.

March 21. From James W. Wallace.

March 22. From R. M. Bucke. Syracuse. CT: Lozynsky, 111-12.

March 23. From R. M. Bucke. Syracuse. ст: Lozynsky, 112.

March 23. From Ellen M. O'Connor.

March 24. From R. M. Bucke. Syracuse. Cт: Lozynsky, 113.

March 25. From Thomas Bailey Aldrich.

March 25. From R. M. Bucke. Syracuse. CT: Lozynsky, 114.

March 26. From R. M. Bucke. Syracuse. Ст: Lozynsky, 115.

March 26. From Ellen M. O'Connor.

March 27. From R. M. Bucke. Syracuse. CT: Lozynsky, 115-16.

March 27. From Ellen M. O'Connor.

March 27. From Ada H. Spaulding.

March 27. From the Photo Engraving Office (acknowledging receipt of $\$ 10.50$ from WW). 
March 27-28. From Edmund Clarence Stedman. ст: Life and Letters of Edmund Clarence Stedman, 2:120-22.

March 28. From R. M. Bucke. Syracuse. CT: Lozynsky, 116.

March 28. From John Burroughs. University of Kansas. ст: Traubel, 4:448-49.

[March 28]. From W. S. Kennedy.

March 29. From Ellen M. O'Connor. Syracuse. ст: Traubel, 4:451.

March 30. From R. M. Bucke. Syracuse. CT: Lozynsky, 116-17.

March 31. From Robert Pearsall Smith.

April 1. From R. M. Bucke. Syracuse. Ст: Lozynsky, 118.

April 2. From R. M. Bucke. Syracuse. ст: Lozynsky, 118-19.

April 3. From R. M. Bucke. Syracuse. ст: Lozynsky, 119.

[April 3]. From Hamlin Garland. ст: Traubel, 4:491-92; $W W Q R$, 5, no. 3 (Winter 1988):10-11.

April 4. From James Gordon Bennett. Yale.

April 4. From R. M. Bucke. Syracuse. ст: Lozynsky, 119-20.

April 4. From Henry A. Holmes, a composer (ordering books).

April 6. From R. M. Bucke. ст: Lozynsky, 120.

April 7. From William W. Payne, of the Chicago Evening fournal, (enclosing his review of November Boughs).

April 8. From W. S. Kennedy.

April 9. From R. M. Bucke. ст: Lozynsky, 120-21.

April 9. From D. H. Kenaga.

April 12. From R. M. Bucke. ст: Lozynsky, 121.

April 13. From R. M. Bucke. ст: Lozynsky, 121-22.

April 14. From R. M. Bucke. ст: Lozynsky, 122.

[April 14?]. From Karl Knortz.

April 18. From Hannah Heyde. Trent.

April [18?]. From W. S. Kennedy.

April 22-24. From Ernest Rhys.

April 24. From R. M. Bucke. ст: Lozynsky, 123.

[April 24?]. From W. S. Kennedy.

April 24. From Ellen M. O'Connor.

April 27. From Will Carleton (ordering a book).
April 28. From Alice Hicks Van Tassel, great grandniece of Elias Hicks (acknowledging receipt of November Boughs).

April 28. From W. S. Kennedy.

April 30. From Ellen M. O’Connor.

May 6. From W. S. Kennedy.

May 9. From Ellen M. O’Connor.

May 9. From James L. Sill (concerning O'Connor's death).

May 10. From R. M. Bucke. ст: Lozynsky, 124.

May 10. From Mary Smith Costelloe. ст: Smith Alumnae Quarterly (February 1958): 88.

May 11. From John Burroughs. ст: Traubel, 5:179-80.

May 11. From George Hall, an English curate and "a humble and obscure lover of yours" (enclosing an article on WW).

May 11-12. From W. S. Kennedy.

May 12. From John B. Barnhill (requesting a manuscript relating to Tolstoy and Sir Walter Scott).

May 12. From R. M. Bucke. cT: Lozynsky, 124-25.

May 13. From R. M. Bucke. ст: Lozynsky, 125-26.

[May 13]. From W. S. Kennedy.

May 14. From Hannah Heyde. LC.

May 14. From Fred S. Ryerson (concerning O'Connor's death).

May 15. From R. M. Bucke. cT: Lozynsky, 126-27.

May 16. From W. S. Kennedy.

May 18. From R. M. Bucke. cr: Lozynsky, 127-28.

May 18. From Edward Carpenter. Ст: Traubel, 5:256.

May 18. From W. S. Kennedy.

May 19. From an unsigned Australian admirer.

May 21. From J. W. Wallace.

May 22. From George W. Childs. PT: Camden's Compliment to Walt Whitman, 64.

[May 23]. From W. S. Kennedy. ст: Traubel, 5:221.

May 24. From Laurence Galimberti, a young Italian laborer.

[May 24]. From W. S. Kennedy.

May 27. From Julius Chambers. ст: Camden's Compliment to Walt Whitman, 67. 
May 28. From R. M. Bucke. ст: Lozynsky, 128.

May 28. From Hamlin Garland. ст: $W W Q R, 5$, no. 3 (Winter 1988): 11.

May 28. From Henry Latchford, of the Chicago Evening Fournal (birthday greetings).

May 30. From Richard J. Hinton. PT: Camden's Compliment to Walt Whitman, 68.

June 1. From W. J. O'Reardon (birthday greetings).

June 1. From Milford C. Reed, a Civil War veteran. Ст: Shively (2), 172-73.

June 2. From R. M. Bucke. ст: Lozynsky, 129.

June 3. From R. M. Bucke. ст: Lozynsky, 129-30.

June 3. From W. S. Kennedy.

June 4. From R. M. Bucke. ст: Lozynsky, 130-31.

June 5. From R. M. Bucke. ст: Lozynsky, 131.

June 7. From Charles L. Heyde. Trent. Ст: Gohdes and Silver, 229-30 (dated "June 89").

June 9. From R. M. Bucke. ст: Lozynsky, 131-32.

[June 10?]. From Elizabeth A. Cottell, an English admirer.

June 12. From W. S. Kennedy.

June 15. From R. M. Bucke. Ohio Wesleyan. ст: Lozynsky, 132.

June 16. From Eduard Bertz. ст: Traubel, 5:330-31.

June 16. From R. M. Bucke. ст: Lozynsky, 133.

June [18?]. From William Carey. ст: Traubel, 5:305.

June 26. From Edward Dowden.

[June?]. From Hamlin Garland. ст: $W W Q R, 5$, no. 3 (Winter 1988): 12 .

[June?]. From Hamlin Garland. ст: $W W Q R, 5$, no. 3 (Winter 1988):12-13.

July 3. From Ellen M. O'Connor.

[July 5]. From R. M. Bucke. Location unknown. PT: Traubel, 5:373, and see Lozynsky, 134.

July 8. From Rudolf Schmidt.

July 9. From W. S. Kennedy.

July 12. From John Burroughs.
[July 12]. From R. M. Bucke. Location unknown. PT: Traubel, 5:366, and see Lozynsky, 134.

July 12. From A. Gardner(?).

July 13. From Charles W. Eldridge.

July 14. From R. M. Bucke. ст: Lozynsky, 135.

July 20-22. From Eduard Bertz.

July 21. From Charles W. Sparkes, an English priest.

[July 22?]. From R. M. Bucke. Location unknown. PT: Traubel, 5:387, and see Lozynsky, 135.

[July 24]. From W. S. Kennedy.

[July 25]. From W. S. Kennedy.

August 1. From Ellen M. O'Connor.

August 3. From Walter B. Whitman, a cadet at Annapolis.

August 4. From R. M. Bucke. ст: Lozynsky, 136.

August 4. From W. S. Kennedy.

August 13. From Robert Pearsall Smith.

August 14. From Ernest Rhys.

August 25. From Henry M. Alden.

August 25. From R. M. Bucke. CT: Lozynsky, 136.

August 27. From John Burroughs.

August 28. From John Oliver (protesting WW's description of religion as "bloodless").

September 1. From O. W. True, a somewhat incoherent admirer.

September 3. From R. M. Bucke. ст: Lozensky, 137.

September 5. From W. S. Kennedy.

September 9. From R. M. Bucke. ст: Lozensky, 137-38.

September 11. From Ernest Rhys.

September 12. From Sir Edwin Arnold.

September 12. From Ellen M. O'Connor.

September 15. From W. S. Kennedy.

September 16. From Thomas Donaldson.

September 19. From the Reverend J. Leonard Corning (thanking WW for photographs).

September 19. From John Foord, editor of Harper's Weekly.

September 19. From Fanny M. Grundy, an English admirer.

September 20. From R. M. Bucke. ст: Lozensky, 138. 
September 21. From Mary A. Fisher (requesting WW to give a reading for the benefit of the Home-Hotel Association for needy authors and artists).

September 21. From Susan Stafford.

September 25. From Marjorie Cook, an eleven-year-old girl.

September 25. From Harper \& Brothers (sending the Fifth Reader).

September 26. From Ellen M. O'Connor.

September 28. From R. M. Bucke. ст: Lozynsky, 138-39.

October 1. From Louis S. Kelley (autograph request).

October 3. From W. S. Kennedy.

October 8. From R. M. Bucke. ст: Lozynsky, 140; Traubel, 6:57-58.

October 8. From Charles W. Eldridge.

October 8. From Laura Daintrey. Location unknown. Ст. Traubel, 6: 75.

[October 10]. From W. S. Kennedy.

October 10. From William P. McKenzie, a young admirer who sent his first book.

October 11. From A. K. McIlhaney, a school principal ("planting a tree and naming it in honor of you").

October 13. From Robert Pearsall Smith.

October 14. From Walter Delaplaine Scull, an English artist.

October 15. From W. S. Kennedy.

October 16. From Richard E. Labar, a young admirer.

October 18. From R. M. Bucke. CT: Lozynsky, 140-41; Traubel, 6:77.

October 18. From W. S. Kennedy.

October 23. From Ernest Rhys.

October 24. From Henry M. Alden. Lion.

October 25. From R. M. Bucke. cT: Lozynsky, 141-42.

October 26. From Mary Smith Costelloe.

October 27. From W. S. Kennedy.

October 27. From James W. Wroth (noting receipt of WW's photograph).

October 29. From R. M. Bucke. ст: Lozynsky, 142-43.

October 30. From R. M. Bucke. cт: Lozynsky, 143.

October. From Charles L. Heyde. Trent.

[October?]. From Hamlin Garland.

November 5. From R. M. Bucke. ст: Lozynsky, 144.

November 5. From W. S. Kennedy.
November 6. From R. M. Bucke. ст: Lozynsky, 144.

November 8. From R. M. Bucke. ст: Lozynsky, 145.

November 8. From Ellen M. O'Connor.

November 9. From Alys Smith.

November 10. From Jessie Louisa Whitman.

November 10. From T. W. H. Rolleston.

November 18. From Charles Aldrich (requesting an autographed copy of " $\mathrm{O}$ Captain! My Captain!” for the Iowa State Library).

November 20. From S. H. Grey, a Camden lawyer (acknowledging receipt of books).

November 20. From Ellen M. O'Connor.

November 27. From Caroline K. Sherman (sending a clipping of her article on Carpenter).

November 29. From Ellen M. O'Connor.

December 3. From R. M. Bucke. CT: Lozynsky, 146.

December 3. From S. S. McClure (requesting WW to comment on "To lift, how little howsoe'er, the hearts of toilers struggling here").

December 7. From Ernest Rhys.

December 8. From R. M. Bucke. ст: Lozynsky, 146-47.

December 9. From John Addington Symonds. ст: Schueller and Peters, 3:42426.

December 13. From Harrison S. Morris (concerning his translation of Sarrazin).

December 15. From Dr. S. Weir Mitchell.

December 17. From John Burroughs.

December 18. From James W. Wroth (Christmas greetings).

December 20. From R. M. Bucke. ст: Lozynsky, 147.

December 20. From William H. Duckett (requesting a loan). ст: Shively (1), 184.

December 21. From Ellen M. O'Connor.

December 22. From R. M. Bucke. ст: Lozynsky, 148.

December 24. From R. M. Bucke. ст: Lozynsky, 148-49.

December 24. From J. B. Wood, of Harleigh Cemetery.

December 24. From Edward Wilkins.

December 27. From Charles L. Heyde. Trent. 
December 27. From W. S. Kennedy.

December 30. From Elizabeth Porter Gould (expressing regret that her verses were not included in Camden's Compliment to Walt Whitman).

December 31. From Dr. John Johnston and J. W. Wallace. Typescript in Bolton.

[December 31]. From F. W. Rean, an admirer.

December. From Charles L. Heyde. Trent.

\section{0}

January 1. From Charles L. Heyde. Trent.

January 2. From Charles L. Heyde. Trent.

January 4. From Alys Smith.

January 4. From Ada H. Spaulding.

January 5. From R. M. Bucke. ст: Lozynsky, 150.

January 6. From Charles L. Heyde. Trent.

January 6. From W. S. Kennedy.

January 6. From James Matlack Scovel (concerning an article about WW by William R. Bates for the Chicago Tribune).

January 7. From R. M. Bucke. Cт: Lozynsky, 150-51.

January 7. From William T. Stead.

January 10. From R. M. Bucke. cT: Lozynsky, 151.

January 12. From R. M. Bucke. ст: Lozynsky, 151-52.

January 14. From Dana Estes, for the Boston Browning Society. Feinberg:Lc.

January 16. From R. M. Bucke. ст: Lozynsky, 152-53.

January 16. From John Swinton. CT: $A L$, 39 (1968): 550-51.

January 17. From R. M. Bucke. ст: Lozynsky, 153.

January 25. From R. M. Bucke. cT: Lozynsky, 154.

January 26. From W. S. Kennedy.

January 29. From R. M. Bucke. CT: Lozynsky, 154-55.

February 1. From George E. Sears (noting that he found "Ambition" in Brother Fonathan).
February 3. From Mary Smith Costelloe. PT: Smith Alumnae Quarterly (February 1958): 88.

February 4. From R. M. Bucke. ст: Lozynsky, 156.

February 7. From Charles L. Heyde. Trent.

February 8. From S. H. Morse.

[February 12]. From W. S. Kennedy.

February 12. From Jacques Reich.

February 12. From Carrie E. Worth (thanking WW for writing to her son).

February 13. From Ellen M. O'Connor.

February 13. From George Rush Jr.

February 14. From Charles L. Heyde. Trent.

February 16. From R. M. Bucke. ст: Lozynsky, 156-57.

February 17. From R. M. Bucke. ст: Lozynsky, 157-58.

February 20. From Mrs. Katherine J. Dowling, a school teacher who asked WW to identify a quotation. Formerly in the Estelle Doheny Collection, St. John's Seminary.

February 21. From James Knowles.

February 24. From Lavina F. Whitman (thanking WW for permitting her visit).

February 27. From John Burroughs. PT: Barrus, 291.

March 6. From R. M. Bucke. ст: Lozynsky, 159.

March 9. From Maurice M. Minton, for The Illustrated American.

[March 12]. From Albert Waldo Howard, a grateful poor poet.

March 12. From W. S. Kennedy.

March 12. From Bernard O'Dowd. ст: Overland, 23 (April 1962):9-10; McLeod, 19-20.

March 14. From Mary Smith Costelloe.

March 15. From Charles L. Heyde. Trent.

March 16. From R. M. Bucke. ст: Lozynsky, 159-60.

March 21. From George R. Morse (autograph request).

March 27. From R. M. Bucke. ct: Lozynsky, 160-61.

[March 31]. From W. S. Kennedy.

April 2. From Maurice M. Minton. 
April 2. From James Matlack Scovel (requesting an item for the Sunday Philadelphia Times).

April 7. From Charles L. Heyde. Trent.

April 11. From Charles L. and Hannah Heyde.

April 12. From Daniel G. Brinton.

April 14. From Rufus C. Hartranft (asking for manuscripts).

April 14. From R. M. Bucke. ст: Lozynsky, 162.

April 15. From Hamlin Garland. ст: $W W Q R, 5$, no. 3. (Winter 1988):13.

April 15. From Dodd, Mead \& Company.

April 17. From Frank G. Carpenter (requesting information for a newspaper article entitled "How to Reach Three Score and Ten Years of Age").

April 18. From Edward Dowden.

April 18. From Charles F. Wingate (requesting words of comfort for the sorrowing).

[April 20?]. From Hamlin Garland. Location unknown. ст: Traubel, 6:377-78.

April 22. From Charles L. Heyde. Trent. April 24. From Charles L. Heyde. Trent.

April 24. From R. M. Bucke. ст: Lozynsky, 162-63.

April 26. From Ernest Rhys.

April 27. From Ellen M. O'Connor.

April 28. From R. M. Bucke. ст: Lozynsky, 163-64.

April 29. From Joseph M. Stoddart.

May 1. From Mary A. Fisher (sending a dollar or two for a "luxury").

May 3. From Charles L. Heyde. Trent.

[May 3]. From W. S. Kennedy.

May 3. From H. R. Maginley (enclosing poems for comment).

May 4. From John Burroughs.

May 7. From Edward Dowden.

May 7. From Carl Falkenreck, an admirer ("I love you! I claim you for a friend! I hold you tight!").

May 10. From Dr. John Johnston and J. W. Wallace. Typescript in Bolton.

May 13. From R. M. Bucke. cт: Lozynsky, 165.

May 17. From Edward Carpenter.

May 21. From Edmund Clarence Stedman.

May 24. From Ernest Rhys.
May 26. From John Swinton. ст: $A L, 39$ (1968):551.

May 28. From Charles L. Heyde. Trent.

[May 29]. From Hezekiah Butterworth, of The Youth's Companion.

May 29. From Ellen M. O'Connor.

May 30. From Emory S. Foster (sending some lines of poetry).

May 31. From Alfred Carpenter (requesting comment about his brother Edward, "a disciple of your opinions").

June 1. From Ellen M. O'Connor.

June 4. From Mrs. Noble T. Biddle (birthday greetings). LC.

June 4. From H. Buxton Forman.

June 4. From Richard E. Labar.

June 5. From Charles L. Heyde. Trent.

June 5. From R. M. Bucke. ст: Lozynsky, 166.

June 5. From Robert G. Ingersoll.

June 5. From J. C. Sears (acknowledging receipt of books).

June 8. From W. S. Kennedy.

June 9. From Bernard O'Dowd. ст: Overland, 23 (April 1962):10-11; McLeod, 20-23.

June 13. From Joseph B. Gilder.

June 13. From Alys Smith.

June 14. From Edward Sprague Marsh (ordering a book).

June 16. From H. Buxton Forman.

June 16. From Robert G. Ingersoll.

June 19. From R. M. Bucke. ст: Lozynsky, 166-67.

June 19. From W. S. Kennedy.

June 20. From Louisa Drewry.

June 23. From Charles B. Campbell (requesting name of WW's publisher). Yale.

June 25. From Thomas Donaldson.

June 26. From R. M. Bucke. ст: Lozynsky, 167-68.

June 27. From J. W. Wallace.

June 29. From J. W. Wallace. Typescript in Bolton.

June 30. From Ellen M. O'Connor.

July 2. From Harry C. Walsh, of Lippincott's Monthly Magazine.

July 3. From Gabriel Sarrazin.

July 3. From "A girl of the states," a friend of Bertha Johnston.

July 6. From R. M. Bucke. CT: Lozynsky, 169. 
July 9. From W. S. Kennedy.

July 11. From Sidney H. Morse.

July 12. From James Grant Wilson (concerning a portrait for The Cyclopedia of American Biography).

July 12. From Andrew H. Rome.

July 16. From Franklin File.

July 16. From William Payne(?).

July 20. From R. M. Bucke. cT: Lozynsky, 169-70.

July 21. From Charles A. Burkhardt (autograph request). Yale.

July [21] (misdated 23). From W. S. Kennedy.

July 27. From R. M. Bucke. ст: Lozynsky, 170.

July 28. From the New York Morning Fournal. LC.

July 30. From Sylvester Baxter.

July 31. From John Swinton (belated birthday wishes). ст: $A L, 39$ (1968): 552.

August 1. From J. W. Wallace. Typescript in Bolton.

August 3. From John Addington Symonds. ст: Schueller and Peters, 3:48184.

August 5. From Louisa Sterling (recommending electric battery treatment).

August 6. From W. S. Kennedy.

August 11. From Horace Traubel.

August [12?]. From M. J. Cummings, melancholic old former poet.

August 12. From W. S. Kennedy.

August 17. From R. M. Bucke. ст: Lozynsky, 171.

August 18-19. From J. W. Wallace.

August 20-22. From R. M. Bucke. ст: Lozynsky, 172.

August 23. From W. S. Kennedy.

August 24. From R. M. Bucke. ст: Lozynsky, 173.

August 28. From Charles L. Heyde. Trent.

August 28. From R. M. Bucke. ст: Lozynsky, 173-74.

August 28. From Robert Pearsall Smith.

August 28. From J. W. Wallace.

[August?]. From Julia A. J. Perkins (autograph request).

[September 1]. From W. S. Kennedy.
September 1. From Bernard O'Dowd. ст: Overland, 23 (April 1962):12; McLeod, 24-26.

September 2. From R. M. Bucke. ст: Lozynsky, 175.

September 5. From John Addington Symonds. ст: Schueller and Peters, 3:49294.

September 5. From J. W. Wallace.

September 8. From Charles L. Heyde. Trent.

September 9. From Robert M. Sillard (ordering a book).

September 9. From J. W. Wallace. Typescript in Bolton.

September 10. From W. S. Kennedy.

September 11-12. From J. W. Wallace.

September 13. From Dr. John Johnston.

[September 14]. From W. S. Kennedy.

[September 15]. From W. S. Kennedy.

September 16. From Jeannette B. Perry, of Vassar College (enclosing her article about WW for The Home, School and Nation).

September 17. From Edmund J. Baillie (greetings from a disciple).

September 17. From R. M. Bucke. ст: Lozynsky, 175-76.

September 18. From R. M. Bucke. ст: Lozynsky, 176-77.

September 19. From J. W. Wallace.

September 20. From Dr. John Johnston.

September 20. From a Japanese admirer.

September 22. From R. M. Bucke. ст: Lozynsky, 177-78.

September 22. From John H. Johnston.

September 22 "later." From John H. Johnston.

September 23. From Dr. John Johnston and J. W. Wallace.

September 23. From John H. Johnston.

September 24. From R. M. Bucke. ст: Lozynsky, 178.

September 24. From H. Buxton Forman.

September 25. From Joseph B. Gilder.

September 25. From John H. Johnston.

September 27. From Dr. John Johnston.

September 28. From R. M. Bucke. ст: Lozynsky, 178-79.

September 29. From Bernard O'Dowd. Ст: Overland, 23 (April 1962):13-14; McLeod, 27-30.

September 29. From Ernest Rhys. 
September 30. From R. M. Bucke. cт: Lozynsky, 179-80.

October 3. From William H. Rideing, for The North American Review.

October 3. From Logan Pearsall Smith.

October 4. From J. W. Wallace. Typescript in Bolton.

October 5. From Ellen M. O'Connor.

[October 6]. From W. S. Kennedy.

[October 6]. From James Matlack Scovel (concerning an article in Lippincott's).

October 7. From R. M. Bucke. ст: Lozynsky, 181.

[October 8]. From Charles L. Heyde. Trent.

October 8. From L. Morrell (delivering a blanket, gift from Wallace and Johnston).

October 9. From Julius Chambers (planning to attend Ingersoll speech).

October 10. From Edmund J. Baillie.

October 10. From Joseph M. Stoddart.

October 11. From J. E. Reinhalter \& Company, which designed and constructed the mausoleum in Harleigh Cemetery).

October 12. From Robert G. Ingersoll. ст: The Letters of Robert G. Ingersoll (1951), 393.

October 12. From R. M. Bucke. cт: Lozynsky, 182.

October 14. From R. M. Bucke. ст: Lozynsky, 182-83.

October 15. From J. W. Wallace.

October 16. From Roger E. Ingpen, an English admirer.

October 16. From G.E. Steinninger (autograph request).

[October 17]. From W. S. Kennedy.

October 20. From Robert G. Ingersoll. ст: The Letters of Robert G. Ingersoll (1951), 394.

October 21. From Dr. John Johnston.

October 22. From John Dewitt Miller (asking WW to certify his 1855 edition of Leaves of Grass).

October 24. From Frederick Oldach \& Company.

[October 25]. From Charles L. Heyde. Trent.

October 26. From R. M. Bucke. ст: Lozynsky, 183-84.

October 27. From Logan Pearsall Smith.
October 27. From Horace Traubel.

October 29. From R. M. Bucke. ст: Lozynsky, 184.

October 31. From David McKay.

November 1. From Jeannette L. and Joseph B. Gilder. Barrett. ст: The Princeton University Library Chronicle, 3 (November 1941):5-6.

November 2. From Gleeson White (requesting permission to visit).

November 2. From R. M. Bucke. ст: Lozynsky, 185.

[November 3]. From Luther Carlyle Jr., a crank ("Yea!! Be thou trebly accursed!!!!”).

November 3. From W. S. Kennedy.

[November 5]. From Charles L. Heyde. Trent. ст: Gohdes and Silver, 231-32.

November 9. From R. M. Bucke. ст: Lozynsky, 185-86.

November 9. From William F. Jackson (expressing admiration for "Old Poets").

November 10. From W. S. Kennedy.

November 11. From R. M. Bucke. ст: Lozynsky, 186-87.

November 11. From William Ingram.

November 14. From Robert Pearsall Smith.

November 15. From R. M. Bucke. ст: Lozynsky, 187-88.

November 15. From Dr. John Johnston.

November 18. From R. M. Bucke. ст: Lozynsky, 188.

November 18. From J. W. Wallace.

November 21. From Joseph $M$. Stoddart.

November 22. From R. M. Bucke. ст: Lozynsky, 188-89.

November 23. From R. M. Bucke. ст: Lozynsky, 189.

November 24. From Bernard O'Dowd. ст: Overland, 23 (April 1962):14-15; McLeod, 30-31.

November 24. From Mrs. Talcott Williams (inviting WW to meet Miss Gale, an actress).

November 27. From Katherine Hardy, a gushing admirer.

November 28. From Mary Smith Costelloe. ст: Smith Alumnae Quarterly (February 1958): 88.

November 28. From Edmund Mercer, an English admirer. 
November 29. From Dr. John Johnston.

November 30. From R. M. Bucke. ст: Lozynsky, 190.

[November]. From Talcott Williams.

[November]. From Charles McIlvaine.

December 1. From Horace Tarr, for The Engineering News (concerning the obituary of Thomas Jefferson Whitman).

December 2. From Jeannette L. and Joseph B. Gilder.

December 2. From B. O. Flower, for The Arena.

December 3. From Charles L. Heyde. Trent.

December 3. From R. M. Bucke. ст: Lozynsky, 191.

December 3. From Dr. John Johnston.

December 3. From Susan Stafford.

December 5. From Talcott Williams.

December 6. From G. Stanley Ellis, an admirer. Yale.

December 8. From Charles L. Heyde. Trent.

December 10. From William T. Stead.

December 11. From Edward Carpenter.

[December 12]. From Charles L. Heyde.

December 12. From R. M. Bucke. ст: Lozynsky, 191-92.

December 12. From Ellen M. O'Connor.

December 12. From Thomas E. Vale, an English admirer.

December 12. From J. W. Wallace. Typescript in Bolton.

December 13. From Dr. John Johnston.

December 13. From Horace Tarr, for The Engineering News.

December 16. From William H. Rideing, for The North American Review.

December 16. From J. W. Wallace.

December 16. From R. M. Bucke. ст: Lozynsky, 192.

December 18. From Gabriel Sarrazin.

December 19. From R. M. Bucke. ст: Lozynsky, 192-93.

December 19. From E. D. Meier (thanking WW for material about his brother Jeff).

December 19. From J. W. Wallace.

[December 20]. From Thomas Hutchinson (requesting an autographed book).

December 20. From Dr. John Johnston.

December 22. From R. M. Bucke. ст: Lozynsky, 193.
December 23. From R. M. Bucke. ст: Lozynsky, 194.

December 23. From Bernard O'Dowd. ст: Overland, 23 (April 1962):15-16; McLeod, 32-33.

December 24. From Charles L. Heyde.

December 26. From F. Baiugrim (acknowledging receipt of a book).

December 27. From Dr. John Johnston.

December 27. From J. W. Wallace.

December 28. From W. S. Kennedy.

December 29. From Charles L. Heyde. Trent.

December 29. From Logan Pearsall Smith.

December 29. From Eva Stafford.

December 31. From Tracy Robinson (New Year's greetings).

[December 31]. From F. W. Rean.

[December]. From W. S. Kennedy.

December. From William Randolph Hearst, for the San Francisco Examiner (asking WW to predict the most important event in 1891).

[1890?] From A. Edward Newton. Yale.

[1890?]. From J. H. Tennant(?) (wanting advice about earning money to go to college). Yale.

[1890?]. From F. Townsend Southwick. LC.

[1890?]. From Alma Calder Johnston (concerning the marriage of her stepdaughter).

\section{1}

January 1. From Elizabeth R. Coffin, an admirer.

[January 1]. From John W. Wroth (New Year's greetings).

January 2. From J. W. Wallace.

January 2. From Ellen M. O'Connor.

January 3. From R. M. Bucke. ст: Lozynsky, 195.

January 3. From Dr. John Johnston.

January 5. From R. M. Bucke. ст: Lozynsky, 195-96.

January 5. From Joseph M. Stoddart.

January 6. From Depuis Macellus Clay (acknowledging receipt of book).

January 6. From J. W. Wallace.

January 6-7. From Dr. John Johnston. 
January 9. From Henry Clapp Jr. (New Year's greetings).

[January 9]. From W. S. Kennedy.

January 9-10. From J. W. Wallace and Dr. John Johnston.

January 10. From The North American Review.

January 12. From R. M. Bucke. ст: Lozynsky, 196-97.

January 12. From W. S. Kennedy.

January 12. From Annie Nathan Meyer, for the Regular Free Library Society (requesting a valentine for a benefit).

January 12. From G. Matthew Jr. (autograph request).

January 13. From Dr. John Johnston.

January 13. From Joseph $M$. Stoddart.

January 14. From the Editors, The Youth's Companion.

January 15. From Joseph B. Gilder.

January 16. From R. M. Bucke. ст: Lozynsky, 197-98.

January 16. From J. W. Wallace.

January 16. From Francis Wilson, the actor.

January 17. From Dr. John Johnston.

January 18. From R. M. Bucke. ст: Lozynsky, 198.

January 19. From Edmund J. Baillie (acknowledging receipt of newspapers and slips).

January 20. From Dr. John Johnston.

January [21]. From Dr. John Johnston.

January 22. From R. M. Bucke. ст: Lozynsky, 198-99.

January 23. From J. W. Wallace.

January 24. From R. M. Bucke. ст: Lozynsky, 199-200.

January 25. From Williamina H. DeJoe (autograph request). LC.

January 27. From an unidentified Scottish writer (concerning a Burns Exhibition in Glasgow).

January 28. From R. M. Bucke. ст: Lozynsky, 200.

January 29. From Laura Lyon White, of The Overland.

January 31. From John Dewitt Miller.

January. From Dr. John Johnston. Typescript in Bolton.

January. From May F. Johnston.

[January?]. From Gustav A. Roedell (autograph request).
February 1. From Bertha Johnston.

February 1. From W. S. Kennedy.

February 2. From R. M. Bucke. ст: Lozynsky, 201.

February 2. From Helen E. Price.

February 2. From Wallace Wood.

February 3. From Havelock Ellis.

February 4. From R. M. Bucke. ст: Lozynsky, 201-2.

February 6. From R. M. Bucke. ст: Lozynsky, 202.

February 6. From Dr. John Johnston.

February 6. From J. W. Wallace.

February 7. From R. M. Bucke. ст: Lozynsky, 202-3.

February 7. From Joseph $M$. Stoddart.

February 8. From R. M. Bucke. ст: Lozynsky, 203-4.

February 9. From R. M. Bucke. ст: Lozynsky, 204.

[February 10?]. From W. S. Kennedy.

February 10. From J. W. Wallace.

February 12. From R. M. Bucke. ст: Lozynsky, 205.

February 14. From R. M. Bucke. ст: Lozynsky, 205-6.

February 14. From E. E. P. Holland (requesting the address of the Gilchrists).

February 14. From H. J. Maywood (enclosing a poetic tribute to WW).

February 15. From Susan Stafford.

February 16. From William T. Stead.

February 17. From John Burroughs.

February 17. From J. W. Wallace.

[February 18]. From W. S. Kennedy.

February 19. From R. M. Bucke. ст: Lozynsky, 206-7.

February 20. From R. M. Bucke. ст: Lozynsky, 207.

February 20. From J. W. Wallace.

February 21. From Dr. John Johnston.

February 22. From R. M. Bucke. ст: Lozynsky, 208.

February 22. From Mrs. J. S. Harris (autograph request). Trent.

February 22. From Margrave Kenyon, an English quack.

February 25. From R. M. Bucke. ст: Lozynsky, 208-9.

February 26. From R. M. Bucke. ст: Lozynsky, 209.

February 27-28. From Dr. John Johnston. 
March 1. From R. M. Bucke. cT: Lozynsky, 210.

March 2. From Fred T. Cozzens.

March 3. From R. M. Bucke. ст: Lozynsky, 210-11.

[March 3]. From James Matlack Scovel.

March 5. From Ellen M. O'Connor.

March 6. From R. M. Bucke. cт: Lozynsky, 211.

March 6. From J. W. Wallace. Typescript in Bolton.

March 8. From R. M. Bucke. ст: Lozynsky, 211-12.

March 8. From Mary A. Jordan, an instructor at Smith College (recalling that as a child she saw WW in Washington).

March 8. From Lucy L. Trautwine (concerning Clifford Harrison's letter which she forwarded to WW).

March 9. From Henry S. Tuke (ordering a book).

March 10. From R. M. Bucke. ст: Lozynsky, 212.

March 11. From Dr. John Johnston.

March 11. From Joseph M. Stoddart.

March 13. From Charles W. Hanley, a sailor.

March 13-14. From J. W. Wallace.

[March 14?]. From Charles L. Heyde. Trent.

March 14. From W. S. Kennedy.

March 15. From Wallace Wood, of the New York Herald. ст: The American Book Collector, 11 (May 1961): 30.

March 16. From Henry M. Alden (concerning the printing of "Death's Valley").

March 16. From M. H. Spielmann (acknowledging receipt of The Conservator).

March 17. From J. W. Wallace. Typescript in Bolton.

March 19. From J. W. Wallace.

March 19. From Jennie Wren (enclosing a copy of The Doll's Dressmaker).

March 20-21. From Dr. John Johnston.

March 22. From R. M. Bucke. ст: Lozynsky, 213.

[March 22?]. From Charles L. Heyde. Trent.

March 24. From R. M. Bucke. ст: Lozynsky, 214.
March 27. From R. M. Bucke. cT: Lozynsky, 214-15.

March 27. From Dr. John Johnston.

March 27. From J. W. Wallace.

March 29. From Ignatius Donnelly.

March 30. From R. M. Bucke. cT: Lozynsky, 215-16.

March 31. From R. M. Bucke. ст: Lozynsky, 216-17.

[March?]. From W. S. Kennedy.

[March?] Friday. From W. S. Kennedy.

April 1. From Dr. John Johnston.

April 2. From Henry B. Binns, the future biographer.

April 3. From R. M. Bucke. ст: Lozynsky, 218.

April 3. From George W. Childs.

April 3. From J. W. Wallace.

April 4. From Dr. John Johnston.

April 4. From Talcott Williams (introducing Miss Belghannie).

April 6. From David McKay. Mrs. Charles Cridland.

April 9-11. From J. W. Wallace.

April 10. From Will Carleton (introducing William Smith).

[April 11]. From Logan Pearsall Smith.

April 13. From R. M. Bucke. ст: Lozynsky, 218-19.

April 13. From Clare Reynolds (enclosing payment for books).

April 13-14. From J. W. Wallace.

April 14. From R. M. Bucke. ст: Lozynsky, 219-20.

April 14-15. From Dr. John Johnston.

April 16. From R. M. Bucke. ст: Lozynsky, 220.

[April 16]. From Elizabeth Porter Gould (acknowledging receipt of photograph).

April 17. From John White Alexander.

April 17. From Raymond Blathwayt (requesting an interview for Pall Mall $G a$ zette).

April 17. From J. W. Wallace. Typescript in Bolton.

April 19. From R. M. Bucke. ст: Lozynsky, 220-21.

April 21. From J. W. Wallace. Typescript in Bolton.

April 22. From Thomas Donaldson (discussing his study of George Catlin).

April 24. From R. M. Bucke. ст: Lozynsky, 221-22. 
April 25. From Julius Chambers.

April 25. From Ellen M. O’Connor.

April 27. From J. E. Reinhalter \& Company.

April 28. From J. W. Wallace. Typescript in Bolton.

April 30. From R. M. Bucke. ст: Lozynsky, 222.

April 30. From J. W. Wallace.

April. From Charles L. Heyde. Trent.

[May 1]. From W. S. Kennedy.

May 2. From Eli Shore, a youthful admirer.

May 2-3. From R. M. Bucke. ст: Lozynsky, 223-24.

[May 5?]. From Raymond Blathwayt (repeating his request for an interview).

May 5. From R. M. Bucke. ст: Lozynsky, 224.

May 6. From Dr. John Johnston.

May 7. From R. M. Bucke. ст: Lozynsky, 224-25.

May 7. From H. Buxton Forman (birthday greetings).

May 7. From J. W. Wallace. Typescript in Bolton.

May 8. From Therese B. H. Brown, an ecstatic admirer.

May 9. From Leonard M. Brown (regretting that he cannot send his annual gift).

May 11. From R. M. Bucke. cт: Lozynsky, 225.

May 12. From Isabella O. Ford (acknowledging receipt of a book).

May 12. From W. S. Kennedy.

May 12. From Calvin B. Knerr (sending payment for a book).

May 12. From J. W. Wallace. Typescript in Bolton. PT: The Walt Whitman Birthplace Bulletin, 3 (January 1960):19.

May 13. From Dr. John Johnston.

May 14. From Alfred Tennyson.

May 16. From Dr. John Johnston.

May 16. From J. W. Wallace.

May 17. From R. M. Bucke. ст: Lozynsky, 225-26.

May 18. From C. H. Greene, an elderly admirer.

May 19. From Melville Philips.

May 19. From J. W. Wallace. Typescript in Bolton.

May 19-20. From Dr. John Johnston.
May 20. From R. M. Bucke. ст: Lozynsky, 226.

May 20. From Edward Carpenter.

May 21. From Charles W. Eldridge.

[May 21]. From W. S. Kennedy.

May 21. From Melville Philips.

May 22. From J. W. Wallace.

May 23. From Dr. John Johnston.

May 25. From R. M. Bucke. ст: Lozynsky, 227.

May 25. From F. A. Hilliard (requesting an autographed Leaves of Grass). Yale.

May 26. From Mrs. Noble T. Biddle (birthday greetings).

May 26. From R. M. Bucke. ст: Lozynsky, 227-28.

May 26-27. From J. W. Wallace and Dr. John Johnston.

May 28. From J. W. Wallace.

May 30. From Dr. John Johnston.

May 31. From Ellen M. O'Connor.

June 1. From Charles L. Heyde. Trent.

June 2. From T. M. Prentiss, a brother of a soldier. (see $P W, 107$ ).

June 2. From F. Townsend Southwick (requesting permission to visit).

June 2. From J. W. Wallace.

June 3. From Dr. John Johnston.

June 4. From Charles L. Heyde. Trent.

June 5. From J. W. Wallace.

June 6. From Dr. John Johnston.

June 6. From N. F. Smith (autograph request).

[June] 8 (misdated May). From R. M. Bucke. ст: Lozynsky, 228.

June 9. From J. W. Wallace.

June 10. From R. M. Bucke. ст: Lozynsky, 229.

June 10. From Dr. John Johnston.

June 10. From Horace Traubel. ст: Lozynsky, 229-30.

June 11. From R. M. Bucke. ст: Lozynsky, 231.

June 11. From Horace Traubel.

June 11. From J. W. Wallace.

June 11-13. From Dr. John Johnston.

June 12. From Frederick Helmick (asking about the direction of future democratic utterances).

June 13. From Wentworth Dixon.

June 14. From R. M. Bucke. ст: Lozynsky, 231-32. 
June 14. From Cecil Reddie (noting admiration of boys at The New School in England for WW's comradeship).

June 15. From William H. Taylor.

June 16. From R. M. Bucke. ст: Lozynsky, 232.

June 16-[17]. From J. W. Wallace.

June 17. From Dr. John Johnston.

June 18. From Charles L. Heyde. Trent.

June 19. From J. W. Wallace.

June 20. From Dr. John Johnston.

June 21. From R. M. Bucke. ст: Lozynsky, 232-33.

June 23. From R. M. Bucke. ст: Lozynsky, 233-34.

June 23. From J. W. Wallace.

[June 25]. From Charles L. Heyde. Trent.

June 26. From R. M. Bucke. cr: Lozynsky, 234.

June 26. From Joseph M. Stoddart.

June 26. From J. W. Wallace.

June 26-27. From Dr. John Johnston.

June 27. From Charles J. Woodbury.

[June] 28 (misdated July). From R. M. Bucke. CT: Lozynsky, 235.

June 29. From R. M. Bucke. ст: Lozynsky, 235-36.

June 30. From R. M. Bucke. ст: Lozynsky, 236-37.

[June]. From Harrison S. Morris (concerning report of WW's birthday in The Literary World).

[June?]. From Lavinia F. Whitman (regretting that WW was too ill to see her).

June 30-July 1. From J. W. Wallace.

July 1. From R. M. Bucke. ст: Lozynsky, 238.

July 1. From Dr. John Johnston.

July 3. From E. L. Townley (sending a list of biblical passages).

July 3-4. From Dr. John Johnston.

July 4. From W. S. Kennedy.

July 8. From R. M. Bucke. ст: Lozynsky, 238-39.

July 10. From Dr. John Johnston.

July 10. From J. W. Wallace.

July 11. From Gabriel Sarrazin.

July 13. From John Phillips Street.

July 15. From Dr. John Johnston.

July 16. From John Phillips Street.

July 18. From R. M. Bucke. ст: Lozynsky, 239-40.

July 18. From Dr. John Johnston.
July 18. From Dr. John Johnston (this letter marked "copy").

July 21. From Elliott Coues, an admirer.

July 21. From Charles G. Garrison (acknowledging receipt of Good-bye My Fancy).

July 21. From David McKay.

[July 21]. From Edith Surridge, an English admirer.

July 21. From J. W. Wallace.

July 22. From Dr. John Johnston.

July 23. From R. M. Bucke. ст: Lozynsky, 240-41.

July 23. From J. W. Wallace.

July 24-25. From Dr. John Johnston.

July 26. From R. M. Bucke. cr: Lozynsky, 241-42.

July 28-29. From J. W. Wallace.

July 29. From Charles L. Heyde. Trent.

July 29. From Dr. John Johnston.

July 31. From R. M. Bucke. ст: Lozynsky, 242-43.

[July 31?]. From Charles L. Heyde.

[July]. From Dr. L. M. Bingham.

July 31-August 1 . From J. W. Wallace.

[August 1-2?]. From R. M. Bucke. ст: Lozynsky, 244 and note.

[August 1-2?]. From Mary Smith Costelloe. See Lozynsky, 244n.

[August 2]. From Charles L. Heyde. Trent.

August 2. From Dr. John Johnston.

August 3. From Charles L. Heyde. Trent.

August 4. From R. M. Bucke. ст: Lozynsky, 245.

August 4. From John H. Johnston.

August 4. From J. W. Wallace.

August 5. From George Horton, of the Chicago Herald (requesting a poem about forthcoming world's fair).

August 6. From Dr. L. M. Bingham.

August 6-7. From Dr. John Johnston.

August 7-8. From J. W. Wallace.

August 8. From Logan Pearsall Smith.

August 10. From R. M. Bucke. ст: Lozynsky, 246-48.

August 11. From Dr. John Johnston.

August 11. From J. W. Wallace.

August 12. From Charles L. Heyde. Trent.

August 14. From J. W. Wallace.

August 14-15. From Dr. John Johnston. 
August 16. From R. M. Bucke. ст: Lozynsky, 248.

August 19. From Dr. John Johnston.

August 21. From William O. McDowell, for the Pan-Republic Congress Committee (requesting a poem).

August 22. From Dr. John Johnston. [August 22]. From W. S. Kennedy.

August 23. From Charles L. Heyde. Trent.

August 26. From Dr. John Johnston.

August 26. From Ellen M. O’Connor.

August 28. From Ada H. Spaulding.

August 29. From Dr. John Johnston.

August 31. From Bernard O'Dowd. ст: Overland, 23 (April 1962):17-18; McLeod, 38-39.

September 1. From R. M. Bucke. ст: Lozynsky, 249.

September 2. From Dr. John Johnston.

September 3. From R. M. Bucke. ст: Lozynsky, 249.

September 5. From Dr. John Johnston.

September 8. From Charles L. Heyde. Trent.

September 8. From H. Buxton Forman.

September 8-9. From Dr. John Johnston.

September 11. From R. M. Bucke. ст: Lozynsky, 249-50.

September 11. From J. W. Wallace.

September 13. From R. M. Bucke. ст: Lozynsky, 250.

September 13. From J. W. Wallace.

September 14. From the Philadelphia Times (inquiring about date of the Ingersoll lecture).

September 14. From Talcott Williams (suggesting an eye examination).

September 15-16. From Dr. John Johnston.

September 16. From Margaretta and William A. Avery, cousins. LC.

September 16. From R. M. Bucke. ст: Lozynsky, 251.

September 16. From Kenneth Crawford, an admirer.

September 16. From L. Morrell, a seaman on the British Prince (regretting that he was unable to visit WW).

September 16. From J. W. Wallace.

September 17. From J. W. Wallace.

September 18. From R. M. Bucke. cT: Lozynsky, 251-52.
September 18. From J. W. Wallace.

September 19. From Dr. John Johnston.

September 19. From J. W. Wallace.

September 20. From R. M. Bucke. ст: Lozynsky, 252.

September 20. From W. S. Kennedy.

September 20. From J. W. Wallace.

September 21. From J. W. Wallace.

September 22. From Hannah Heyde. Lc.

September 22. From J. W. Wallace.

September 23. From Dr. John Johnston.

September 23. From J. W. Wallace.

September 24. From J. W. Wallace.

September 26. From Dr. John Johnston.

September 26. From Edward Wilkins.

September 27. From R. M. Bucke. ст: Lozynsky, 252-53.

September 27. From J. W. Wallace. Typescript in Bolton.

September 28. From J. W. Wallace.

September 29. From J. W. Wallace.

September 30. From Dr. John Johnston.

September 30. From J. W. Wallace.

October 1. From Werner Bruns (inquiring whether WW was interested in land near Huntington, Long Island).

[October] 2 (misdated September). From R. M. Bucke. ст. Lozynsky, 254-55.

October 2. From James Watt, a Scottish admirer.

October 4. From J. W. Wallace.

October 5. From J. W. Wallace.

October 9. From George E. Sears (concerning Brother fonathan).

October 9. From J. W. Wallace.

October 10. From Dr. John Johnston.

October 10. From J. W. Wallace.

October 11. From J. W. Wallace.

October 12. From Samuel G. Stanley (belated birthday greetings).

October 13. From R. M. Bucke. ст: Lozynsky, 255.

October 13. From Joseph B. Gilder. Berg.

October 13. From John H. Johnston.

October 14. From Dr. John Johnston.

October 14. From J. W. Wallace.

October 17. From R. M. Bucke. ст: Lozynsky, 255-56.

October 17. From H. Buxton Forman (acknowledging receipt of books).

October 17. From Dr. John Johnston.

October 21. From Charles L. Heyde. Trent. 
October 22. From R. M. Bucke. CT: Lozynsky, 256.

October 23. From John Russell Young.

October 24. From Dr. John Johnston.

[October 24]. From Ralph E. Moore (ordering a book).

October 25. From R. M. Bucke. cт: Lozynsky, 256-57.

October 25. From Samuel G. Stanley.

October 26. From Ethel Webling, an English admirer.

October 27. From R. M. Bucke. cт: Lozynsky, 257.

October 27. From J. E. Reinhalter \& Company.

October 28. From Dr. John Johnston.

October 29. From R. M. Bucke. ст: Lozynsky, 257-58.

October 29. From May F. Johnston (concerning Sir Edwin Arnold).

October 30. From W. S. Kennedy.

October 30. From Ida Strauss, a cousin (autograph request).

October 31. From Dr. John Johnston.

October 31. From J. E. Reinhalter \& Company.

October. From Arthur Lynch (sending a book written by a young Australian).

[November 1]. From John Russell Young.

November 3. From R. M. Bucke. CT: Lozynsky, 259.

November 3. From Lavinia F. Whitman (concerning Sir Edwin Arnold).

November 3. From John Russell Young.

November 4. From Mrs. Anna Hatch, an admirer.

November 4. From Dr. John Johnston.

November 4. From Mrs. M. B. Minchen, an admirer.

November 5. From the Postmaster, Delaware County, Ohio.

November 6. From R. M. Bucke. ст: Lozynsky, 259-60.

November 7. From Dr. John Johnston.

November 8. From H. Buxton Forman.

November 8. From Thaddeus Hyatt, a Brooklyn admirer.

November 10. From R. M. Bucke. ст: Lozynsky, 260.

November 11. From Val. Stuart Redden (recalling his meeting with WW in Washington).
November 11. From Josephine Webling (sending a copy of Leaves of Grass to be autographed).

November 14. From R. M. Bucke. ст: Lozynsky, 260-61.

November 14. From Dr. John Johnston.

November 14. From Moses King (sending copy of King's Handbook of the United States).

November 14. From Ellen M. O'Connor.

November 14. From J. W. Wallace.

November 15. From Josephine Webling (acknowledging receipt of autographed book).

November 16. From Dr. L. M. Bingham.

November 16. From R. M. Bucke. ст: Lozynsky, 261.

November 18. From Dr. John Johnston.

November 20. From Edward Carpenter.

November 20. From Dr. John Johnston.

November 20. From J. W. Wallace.

November 21. From R. M. Bucke. ст: Lozynsky, 262.

November 21. From Robert M. Sillard (inquiring about WW's favorite books and authors).

November 22. From Logan Pearsall Smith.

November 23. From The Illustrated American (noting an article of interest to WW in the current issue).

November 24. From R. M. Bucke. ст: Lozynsky, 262-63.

November 24. From W. M. Reeder (sending a barrel of potatoes and cider).

November 24. From Harry L. Dwight (inviting WW to live with him in Friendship in New York).

November 25. From Mrs. Talbot Buckny (recalling the association of her father Jesse Talbot with WW in 1832).

November 25. From Dr. John Johnston.

November 25. From Charles H. Roberts (recalling his meeting with WW years ago).

November 26. From R. M. Bucke. ст: Lozynsky, 263 (misdated November 6).

November 26. From H. Buxton Forman.

November 28. From Dr. John Johnston.

November 28. From J. W. Wallace.

November 30. From John H. Johnston.

November 30. From J. William Lloyd (ordering a book). 
December 1. From J. Armory Knox (sending best wishes).

December 2. From J. William Lloyd (acknowledging receipt of a book).

December 2. From Dr. John Johnston.

December 2. From Serelda G. Thomas (requesting a copy of his writings for review in The Democrat).

December 3. From Helen J. Holcomb (sending a poem).

December 3. From Dr. Daniel Longaker.

December 4-5. From Dr. John Johnston.

December 5. From William Carey, of Century.

December 5. From J. W. Wallace.

December 7. From James Stoddart (ordering a book).

December 8. From R. M. Bucke. ст: Lozynsky, 264.

December 8. From William Carey.

December 9. From J. W. Wallace.

December 11. From R. M. Bucke. ст: Lozynsky, 264.

December 12. From Robert G. Ingersoll.

December 12. From J. W. Wallace.

December 14. From Luther Munday (requesting a few words).

December 15. From J. E. Holdsworth, of the Labour Church in Manchester, England (requesting permission to use WW's poems in a hymn book).

December 16. From Dr. John Johnston.

December 16. From J. W. Wallace.

December 17. From H. Buxton Forman.

December 19. From Edward Carpenter (enclosing $£ 4$ for books).

December 19. From J. W. Wallace.

December 20. From R. M. Bucke. ст: Lozynsky, 265.

December 20. From John Burroughs.

December 20. From Lavinia F. Whitman (expressing hope for his recovery).

December 21. From Edward T. Wood (furnishing medical nostrums).

December 22. From E. J. Smith, an admirer in Tacoma, Washington.

December 22. From Frank and Ellen Webb, who named their child Walter Whitman Webb.

December 23. From Dr. John Johnston.

December 26. From Ada H. Spaulding (best wishes for his recovery).
December 29. From Robert G. Ingersoll. ст: The Letters of Robert G. Ingersoll (1951), 396.

December 30. From Dr. John Johnston.

[1891?]. From Eliza Seaman L. LC.

\section{2}

January 2. From J. W. Wallace.

January 4. From A. C. Benedict (reminiscing about Washington days).

January 5. From Isabel Yeomans Brown, a Canadian admirer.

January 6. From Dr. John Johnston.

January 6. From Mrs. J. L. Pittman, a Brooklyn admirer.

January 7. From Harry E. Boutelle (sending sea weed from Pacific Ocean).

January 9. From W. I. Lincoln, editor of The Photographic Times (concerning the use of a photograph and forwarding a brief biographical account for correction).

[January 12]. From Charles L. Heyde. Trent.

January 12. From H. D. Bush (acknowledging receipt of final edition of Leaves of Grass).

January 17. From Lavinia F. Whitman.

January 21. From Arthur Stedman (introducing William Sharp). Berg.

January 21. From John Swinton (best wishes for his recovery). Ст: $A L, 39$ (1968): 552.

January 24. From Hannah Heyde. Trent. Ст: Gohdes and Silver, 212.

January 26. From Hallam Tennyson.

January 28. From J. W. Wallace.

February 2. From J. W. Wallace.

February [3]. From Elizabeth Porter Gould.

February 6. From Robert G. Ingersoll.

February 9. From Robert G. Ingersoll.

February 16. From J. W. Wallace.

February 17. From Frank Cowan (sending his Poetic Works).

February 17. From Dr. John Johnston.

February 18. From J. W. Wallace.

February 20. From Dr. John Johnston.

February 23. From J. W. Wallace.

February 24. From Wentworth Dixon.

February 24. From Max A. Wright, a friend of Dr. Johnston. 
February 25. From Sam G. Thompson, an English admirer.

February 25. From J. W. Wallace.

February 27. From Dr. John Johnston.

February [29]. From Charles L. Heyde. Trent.

March 1. From Margaretta and William A. Avery.

March 1. From J. W. Wallace.

March 2. From Dr. John Johnston.

[March] 4 (misdated February). From J. W. Wallace.

March 5. From Dr. John Johnston.

March 5. From Fred Wild.

March 8. From Standish James O'Grady (best wishes for recovery).

March 8. From J. W. Wallace.

March 8. From T. W. H. Rolleston.

[March 9]. From George Humphreys.

March 12. From Dr. John Johnston.

March 12. From Byramjie Soralejea (enclosing a poem).

March 12. From J. W. Wallace.

March 14. From Albert Hopkins (about a society devoted to the pansy).

March 15. From Hannah Heyde. LC.

March 15. From J. W. Wallace.

March 16. From Mrs. L. Dillard (hoping for his recovery).

March 16. From Dr. John Johnston.

March 18. From Thaddeus Hyatt.

March 18. From J. W. Wallace. Whitman House, Camden.

March 19. From Dr. John Johnston. Whitman House, Camden.

March 20. From Blake Bigelow, an ardent admirer.

March 21. From Cyrus C. Miller (ordering books).

March 22. From John Hay (requesting the Centennial Edition).

March 22. From J. W. Wallace.

March 23. From Dr. John Johnston.

March 24. From Robert G. Ingersoll.

March 25. From J. W. Wallace.

March 26. From Dr. John Johnston.

\section{UNDATED}

\section{[Arranged Alphabetically]}

June 21. From Henry W. Armstrong (autograph request).
September. From A. W. Bond(?) (autograph request). LC.

From Marie Case (inviting WW to come to see her "little friend. . . . good boy of 20 and myself"). See $D B N, 157$.

April 27. From Oswald Cave (requesting permission to quote a Whitman poem in his lecture series).

September 1. From Christian Kingdom Society (inviting WW to join).

Monday. From Emma Dirngee(?) (autograph request). LC.

From Preston Harrison, a Chicago admirer. LC.

August 12. From Joseph G. Heyn (autograph request).

[March 19]. From Charles L. Hildreth (concerning an article about WW).

May 31. From Bim M. Jordan(?). LC.

[May 10]. From W. S. Kennedy (inviting WW to come to Boston).

From Karl Knortz (concerning a visit from Bucke and the German translation of Leaves of Grass).

July. From Mrs. Main(?) Blood (noting receipt of a picture).

From Dr. Charles McIlvaine (transmitting a poem entitled "Ho! Help God!"). See $D B N, 382 \mathrm{n}, 723$.

From Joaquin Miller (concerning Chicago World Fair).

From Dr. W. E. Mitchell (referring to T. B. Aldrich and Harriet Beecher Stowe). LC.

From R. Rooke Morgan. LC.

December 6. From William Robinson (autograph request).

Monday. From James Matlack Scovel (sending "Power Temperance").

Wednesday. From James Matlack Scovel (requesting pieces for the Philadelphia Times).

Sunday. From James Matlack Scovel (concerning WW's illness).

From Ernest Denton Seybold (a note to WW included in his letter to his father).

August 3. From Elmina D. Slenker (praising WW for proposing much needed "sexual reform"). See $D B N, 323$.

From Marie Wallace, an enthusiastic admirer. Feinberg:Lc. 
January 2. From Lavinia F. Whitman (concerning the Whitman genealogy). LC.

From Mrs. Talcott Williams (presenting a Mrs. Pound Fort?).

Wednesday. From Mrs. Talcott Williams (introducing a Mr. Alexander of Henry Irving's company).
Friday. From Talcott Williams (inviting WW to go on a barge excursion).

Sunday. From Talcott Williams (introducing Ellen Louise Chandler Moulton).

From Bayard Wyman (requesting a photograph and a holograph poem). 